\title{
A simple molecular orbital picture of RIXS distilled from many-body damped response theory
}

\author{
Kaushik D. Nanda ${ }^{a, *}$ and Anna I. Krylov ${ }^{a, \dagger}$ \\ ${ }^{a}$ Department of Chemistry, University of Southern California, Los Angeles, California 90089-0482 \\ * kaushikdnanda@gmail.com (K.D.N.), † krylov@usc.edu (A.I.K.)
}

$A b$ initio calculations of resonant inelastic X-ray scattering (RIXS) rely on the damped response theory, which prevents the divergence of response solutions in the resonant regime. Within the damped response theory formalism, RIXS moments are expressed as sum over all electronic states of the system (SOS expressions). By invoking resonance arguments, these expressions can be reduced to a few terms, an approximation commonly exploited for interpretation of the computed cross sections. We present an alternative approach: a rigorous formalism for deriving a simple molecular orbital picture of the RIXS process from the many-body calculations using damped response theory. In practical implementations, the SOS expressions of RIXS moments are recast in terms of matrix elements between the zero-order wave functions and first-order frequency-dependent response wave functions of the initial and final states, such that the RIXS moments can be evaluated using complex response one-particle transition density matrices (1PTDMs). Visualization of these 1PTDMs connects the RIXS process with the changes in electronic density. We demonstrate that the real and imaginary components of the response 1PTDMs can be interpreted as contributions of the undamped off-resonance and damped near-resonance SOS terms, respectively. By analyzing these 1PTDMs in terms of natural transition orbitals, we derive a rigorous, black-box mapping of the RIXS process into a molecular orbital picture. We illustrate the utility of the new tool by analyzing RIXS transitions in the $\mathrm{OH}$ radical, benzene, para-nitroaniline, and 4-amino-4'-nitrostilbene. These examples highlight the significance of both near-resonance and off-resonance channels. 


\section{INTRODUCTION}

Resonant inelastic X-ray scattering ${ }^{1-5}$ (RIXS) is a two-photon process wherein a resonant $\mathrm{X}$-ray photon is absorbed and another X-ray photon of lower energy is emitted. RIXS is a coherent two-step process that involves photoexcitation of a core electron creating a coreexcited state and simultaneous filling of this core hole by radiative decay of a valence electron, as shown in Fig. 1. Thus, the overall transition is from the ground state to a valence excited state and the difference in the photon energies equals the energy gap between the initial (ground) and the final (usually, valence excited) states of the system.

The RIXS process is often described as a two-step process comprising x-ray absorption and x-ray emission. Owing to its two-photon nature, RIXS transitions obey different selection rules than one-photon transitions. Thus, RIXS provides information complementary to that delivered by x-ray absorption and emission spectroscopies (XAS and XES, respectively). Similar to XAS and XES, RIXS exploits the elemental specificity of x-rays, the compact nature of the core orbitals, and the strong sensitivity to the local environment, bonding pattern around, and oxidation state of a specific atom ${ }^{6}$. RIXS has been used extensively

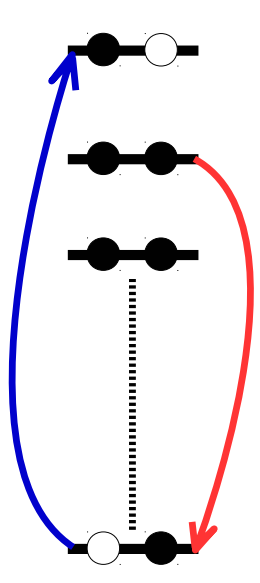

(a)

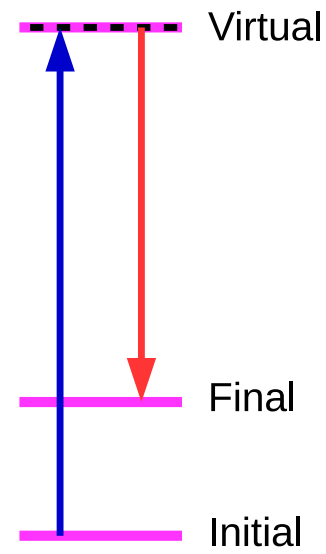

(b)

FIG. 1: Schematic representation of the RIXS process. (a) Molecular orbital picture: one electron is excited to an unoccupied orbital, leaving behind a hole, and another electron is coherently dede-excited, filling the hole. (b) Many-body states' picture: The dashed line depicts the virtual intermediate state, often considered to be a core-excited state resonant with the incoming photon's energy. 
for probing charge-transfer and crystal-field transitions in metal oxides ${ }^{4,5}$. With the advent of high-brilliance radiation sources, RIXS is now also used to study excited-state nuclear dynamics $^{7}$ and to detect transient species in ultrafast reactions in complex environments ${ }^{8}$.

Theoretical modeling of the RIXS spectra is critical for connecting the measured RIXS spectra with the electronic structure of the molecule. Ultimately, the mechanistic interpretation the spectra hinges on our ability to describe the underlying process in terms of transitions between molecular orbitals.

The orbital picture of one-photon UV-visible (UV-vis), XAS, and XES transitions can be obtained from many-body wave functions by using reduced quantities, such as one-particle transition density matrices (1PTDMs) and natural transition orbitals (NTOs) ${ }^{9-15}$. 1PTDMs contain essential information about electronic transitions needed to compute the observables (e.g., cross sections). NTOs, computed by singular value decomposition (SVD) of 1PTDMs, provide the most compact representation of the transition in terms of the hole and particle states. In contrast to the wave-function amplitudes, NTOs are invariant with respect to all allowed orbital rotations, which makes them insensitive to the basis-set choice. Furthermore, since 1PTDMs and NTOs are directly mapped to the experimental observables, they are observables themselves. Thus, NTOs provide a rigorous and robust framework for wavefunction analysis.

The concept of NTOs has been generalized to two-photon absorption (2PA) transitions, enabling the analysis of the corresponding response 1PTDMs and characteristic 2PA virtual states ${ }^{16}$; to non-Hermitian quantum mechanics, enabling the analysis of the complexvalued 1 PTDMs and transitions involving states in the continuum ${ }^{17}$; and to spinless $1 \mathrm{PT}$ DMs, enabling the analysis of tensorial properties (spin-orbit couplings) and spin-forbidden transitions ${ }^{18}$. Here, we extend the concept of NTOs to RIXS transition moments.

The main challenge in interpreting the RIXS process in terms of molecular orbitals stems from its nonlinear (two-photon) nature. Because of it, the scattering moments are given by cumbersome sum-over-states (SOS) expressions ${ }^{19-21}$ and not by matrix elements between the initial and final states, as in the case of UV-vis, XAS, and XES transitions. This dependence on all electronic states of the system makes the analysis of RIXS moments more difficult than the analysis of one-photon moments (matrix elements of dipole operator between the initial and final states). Furthermore, the RIXS moments are complex-valued and tensors of rank two $(3 \times 3$ matrices $)$, in contrast to the one-dimensional one-photon moments, which 
are real-valued vectors along the three Cartesian coordinates.

Because of its resonant nature, the qualitative picture of the RIXS transition is traditionally derived using approximate few-states models - in particular, a three-states modelinvolving few near-resonant core-excited states along with the initial and final states. In a few-states model, the orbitals involved in the transitions from the initial state to different intermediate states and from these intermediate states to the final state are computed and stitched together to construct the orbital picture of the RIXS process. For example, in the three-states model, the virtual state (see Fig. 1) of the two-photon RIXS process corresponds to the core-excited state for which the XAS peak is resonant with the incoming photon's energy. While being physically justified, such approach involves arbitrariness and is prone to a potential loss of accuracy because it is not always easy to identify the important intermediate states that need to be included in these few-state models. In this approach, the orbital character of the virtual state of the RIXS process is determined by the (somewhat arbitrary) choice of the intermediate states picked in the few-states model. The loss of accuracy can occur when off-resonance channels make non-negligible contributions to the RIXS cross sections.

Here, we overcome these challenges using a novel approach of deriving the mechanistic details of the RIXS transitions by means of NTOs computed directly from the complexvalued damped response 1PTDMs that enter the expressions of RIXS moments. This leads to a rigorous and black-box procedure of mapping the computed scattering moments into molecular orbitals. In contrast to traditional approaches, our scheme does not invoke arbitrary truncation of the SOS expressions and is orbital invariant. We discuss the meaning of the real and the imaginary components of these 1PTDMs and the corresponding NTOs by analyzing RIXS transitions in the $\mathrm{OH}$ radical, benzene, para-nitroaniline (pNA), and 4-amino-4'-nitrostilbene (4A4NS). The pNA and 4A4NS examples illustrate the importance of off-resonance RIXS channels and highlight the advantages of fully analytic calculation and analysis of the RIXS moments over approximate treatments by few-states models. We also illustrate how a quantitative metric for the extent of delocalization of electronic density during the RIXS transition can be computed using these response 1PTDMs. While this approach builds upon our prior work on $2 \mathrm{PA}$ transitions ${ }^{16}$, the novelty lies in the interpretation of complex-valued RIXS 1PTDMs (and their NTOs) instead of the real-valued 2PA 1PTDMs. 


\section{THEORY}

The RIXS scattering moments are given by the Kramers-Heisenberg-Dirac formula as SOS expressions ${ }^{19-22}$ :

$$
M_{g \rightarrow f}^{x y}\left(\omega_{1 x},-\omega_{2 y}, \epsilon\right)=-\sum_{n}\left(\frac{\left\langle\Psi_{f}\left|\mu^{y}\right| \Psi_{n}\right\rangle\left\langle\Psi_{n}\left|\mu^{x}\right| \Psi_{g}\right\rangle}{\Omega_{n g}-\omega_{1 x}-i \epsilon_{n}}+\frac{\left\langle\Psi_{f}\left|\mu^{x}\right| \Psi_{n}\right\rangle\left\langle\Psi_{n}\left|\mu^{y}\right| \Psi_{g}\right\rangle}{\Omega_{n g}+\omega_{2 y}+i \epsilon_{n}}\right)
$$

and

$$
M_{f \rightarrow g}^{x y}\left(-\omega_{1 x}, \omega_{2 y},-\epsilon\right)=-\sum_{n}\left(\frac{\left\langle\Psi_{g}\left|\mu^{x}\right| \Psi_{n}\right\rangle\left\langle\Psi_{n}\left|\mu^{y}\right| \Psi_{f}\right\rangle}{\Omega_{n g}-\omega_{1 x}+i \epsilon_{n}}+\frac{\left\langle\Psi_{g}\left|\mu^{y}\right| \Psi_{n}\right\rangle\left\langle\Psi_{n}\left|\mu^{x}\right| \Psi_{f}\right\rangle}{\Omega_{n g}+\omega_{2 y}-i \epsilon_{n}}\right) .
$$

where $\Omega_{n g}=E_{n}-E_{g}$ and $\omega_{1 x}$ and $\omega_{2 y}$ are the absorbed and emitted frequencies (polarized along $x$ - and $y$-directions), satisfying the RIXS resonance condition,

$$
\omega_{1}-\omega_{2}=\Omega_{f g}
$$

Note that

$$
M_{f \rightarrow g}^{x y}\left(-\omega_{1 x}, \omega_{2 y},-\epsilon\right)=\left(M_{g \rightarrow f}^{x y}\left(\omega_{1 x},-\omega_{2 y}, \epsilon\right)\right)^{*}
$$

where $^{*}$ denotes complex conjugation. In Hermitian theories, the two scattering moments are complex conjugates of each other; however, for coupled-cluster methods, this is not the case $^{20-22} . \epsilon_{n}$ is the inverse lifetime parameter for state $n$. If the lifetimes of all states are infinite $\left(\epsilon_{n}=0 \forall n\right)$, in the case of the RIXS, at least for one state $k$, the SOS has zero denominator $\left(\Omega_{k g}-\omega_{1}=0\right)$. In other words, the RIXS moments have first-order poles at $\Omega_{n g}$ s. Practically, this means that attempts to calculate RIXS moments assuming infinite lifetimes of electronic states result in divergent solutions.

Most theoretical formulations for calculating RIXS moments use empirical non-zero inverse lifetimes for all states, which are assumed to have the same nonzero value $\epsilon^{19-25}$. The introduction of this imaginary phenomenological (damping) parameter $i \epsilon$ brings the poles due to the resonances into the complex plane. The impact of introducing $i \epsilon$ on individual SOS terms depends on whether $\left|\Omega_{n g}-\omega_{1}\right|$ is less or greater than $|\epsilon|$, as explained in Fig. 2. The contribution of the SOS terms that have $\left|\Omega_{n g}-\omega_{1}\right|<|\epsilon|$ (nearly resonant SOS terms) is dominated by their imaginary components. The real components of these terms are smaller than the imaginary components. In particular, for the SOS terms with $\left|\Omega_{n g}-\omega_{1}\right|=0$, the real components are zero and the imaginary components equal $\frac{\left\langle\Psi_{f}\left|\mu^{y}\right| \Psi_{n}\right\rangle\left\langle\Psi_{n}\left|\mu^{x}\right| \Psi_{g}\right\rangle}{\epsilon}$ (or 


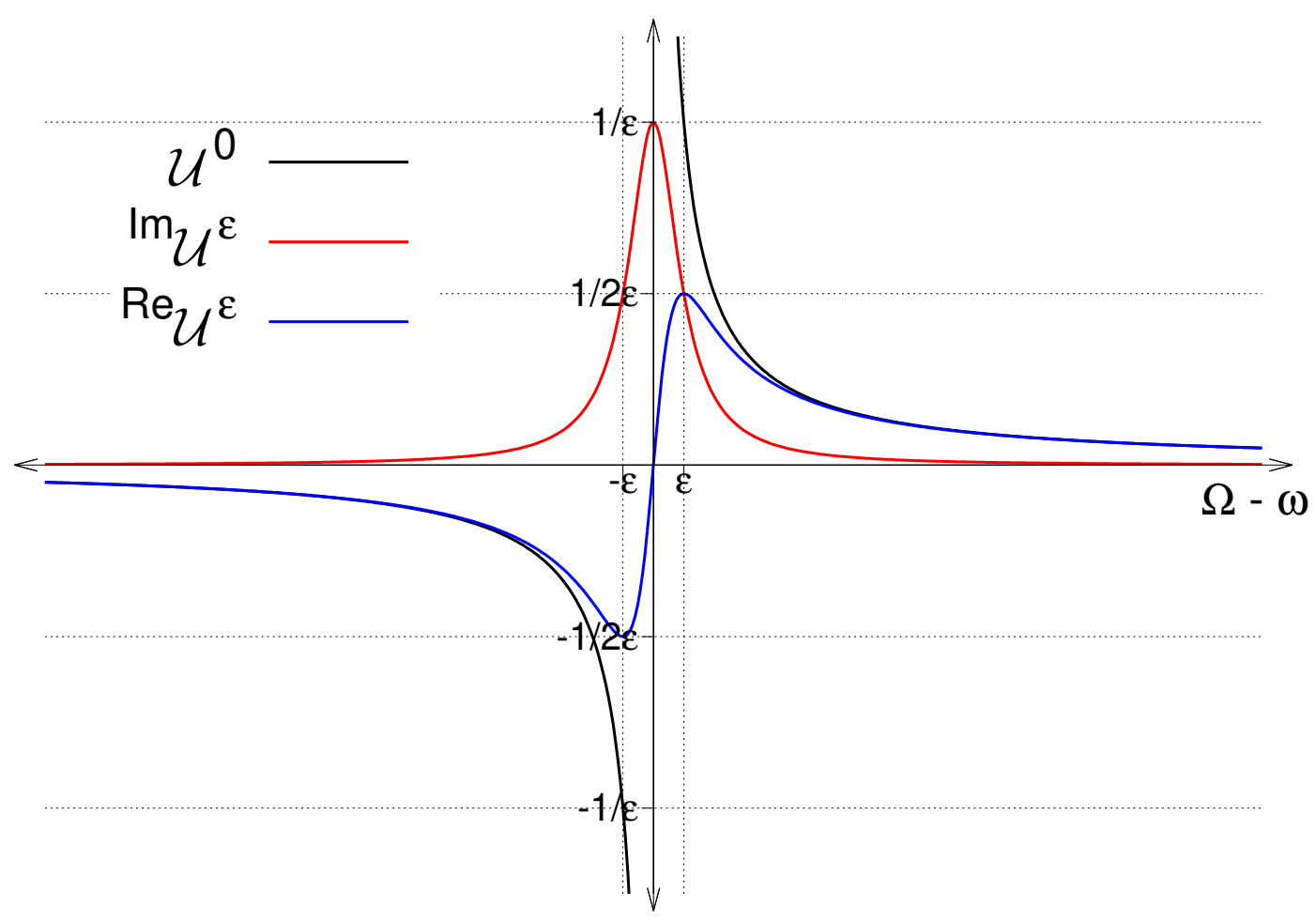

FIG. 2: Impact of the imaginary phenomenological damping $(i \epsilon)$ on the RIXS moments. Individual SOS terms are given by $\frac{\left\langle\Psi_{g}|\mu| \Psi_{n}\right\rangle\left\langle\Psi_{n}|\mu| \Psi_{f}\right\rangle}{\Omega_{n g}-\omega+i \epsilon}=\mu_{g n} \mu_{n f} \mathcal{U}^{\epsilon}\left(\Omega_{n g}-\omega\right)$, where $\mathcal{U}^{\epsilon}\left(\Omega_{n g}-\omega\right)$ is related to the Green's function: $G^{+}(\omega)=\lim _{\epsilon \rightarrow 0^{+}}[H-\omega+i \epsilon]^{-1}=\lim _{\epsilon \rightarrow 0^{+}} \sum_{n}\left|\Psi_{n}\right\rangle \mathcal{U}^{\epsilon}\left(\Omega_{n g}-\omega\right)\left\langle\Psi_{n}\right|$. The poles of $\mathcal{U}$ are also poles of the Green's function. $\mathcal{U}$ can be written as $\mathcal{U}^{\epsilon}(\Omega-\omega)=\left(\frac{1}{\Omega-\omega+i \epsilon}\right)$. The black hyperbola represents $\mathcal{U}$ without the imaginary damping, i.e., $\mathcal{U}^{0}(\Omega-\omega)=\left(\frac{1}{\Omega-\omega}\right)$. When $\Omega-\omega=0$, this function has a pole and is indeterminate. The red and blue curves represent the imaginary and real components of damped $\mathcal{U}, \operatorname{Im} \mathcal{U}^{\epsilon}(\Omega-\omega)=\frac{\epsilon}{(\Omega-\omega)^{2}+\epsilon^{2}}$ and $\operatorname{Re}^{\epsilon}(\Omega-\omega)=$

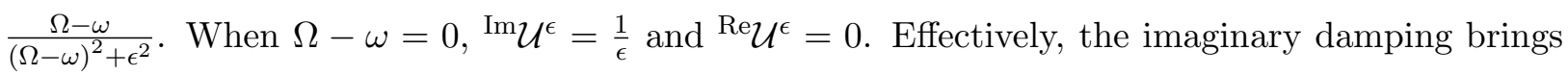
the damped contribution of $\mathcal{U}^{0}$ predominantly into the imaginary component of $\mathcal{U}^{\epsilon}$ for $\Omega-\omega<\epsilon$ (near resonance). For $\Omega-\omega>\epsilon$ (off resonance), the damping has a smaller impact such that $\mathcal{U}^{0}$ and ${ }^{R e} \mathcal{U}^{\epsilon}$ have similar magnitude for large $\Omega-\omega$.

$\left.\frac{\left\langle\Psi_{g}\left|\mu^{x}\right| \Psi_{n}\right\rangle\left\langle\Psi_{n}\left|\mu^{y}\right| \Psi_{f}\right\rangle}{\epsilon}\right)$; thus, the absolute contribution of each of these SOS terms is effectively damped from infinity to a finite value by virtue of $i \epsilon$. On the other hand, the real components are larger than the imaginary ones for the SOS terms for which $\left|\Omega_{n g}-\omega_{1}\right|>|\epsilon|$. In short, damping puts the near-resonance and off-resonance contributions in the imaginary and real components of the RIXS moments, respectively. 
Computing the full set of electronic states for calculating the RIXS moments via Eqs. (1) and (2) is obviously impractical. Often, an approximated (truncated) SOS can provide a qualitatively correct value of the RIXS moment; however, the error introduced due to truncation is difficult to evaluate a priori. Nevertheless, many studies employ such truncations for computing the RIXS moments and other non-linear properties. An alternative, more rigorous strategy involves recasting the SOS expressions into a closed form using damped response theory ${ }^{19-22,26-31}$. By doing so, one circumvents the need to compute the wave functions and energies of all electronic states. Instead, only a handful of response wave functions need to be computed ${ }^{26}$. The RIXS moments computed with the damped response theory approach are formally and numerically equivalent to the full SOS result. This strategy for RIXS calculations has been exploited in the analytic implementations based on algebraic diagrammatic construction ${ }^{19}$, coupled-cluster ${ }^{20,22}$, and equation-of-motion coupled-cluster ${ }^{21}$ methods. Importantly, the damped response theory approach can be formulated in terms of response transition density matrices, which can be exploited to obtain a concise description of the RIXS transition.

Within the damped response theory framework with $\epsilon_{n}=\epsilon \forall n$, Eqs. (1) and (2) are rewritten using Eq. (3) as

$$
\begin{aligned}
M_{g \rightarrow f}^{x y}\left(\omega_{1 x}, \epsilon\right) & =-\sum_{n}\left(\frac{\left\langle\Psi_{f}\left|\mu^{y}\right| \Psi_{n}\right\rangle\left\langle\Psi_{n}\left|\mu^{x}\right| \Psi_{g}\right\rangle}{\Omega_{n g}-\omega_{1 x}-i \epsilon}+\frac{\left\langle\Psi_{f}\left|\mu^{x}\right| \Psi_{n}\right\rangle\left\langle\Psi_{n}\left|\mu^{y}\right| \Psi_{g}\right\rangle}{\Omega_{n f}+\omega_{1 x}+i \epsilon}\right) \\
& =-\left(\left\langle\Psi_{f}\left|\mu^{y}\right| X_{g}^{\epsilon, \omega_{1 x}}\right\rangle+\left\langle\tilde{X}_{f}^{\epsilon, \omega_{1 x}}\left|\mu^{y}\right| \Psi_{g}\right\rangle\right)
\end{aligned}
$$

and

$$
\begin{aligned}
M_{f \rightarrow g}^{x y}\left(\omega_{2 y},-\epsilon\right) & =-\sum_{n}\left(\frac{\left\langle\Psi_{g}\left|\mu^{x}\right| \Psi_{n}\right\rangle\left\langle\Psi_{n}\left|\mu^{y}\right| \Psi_{f}\right\rangle}{\Omega_{n f}-\omega_{2 y}+i \epsilon}+\frac{\left\langle\Psi_{g}\left|\mu^{y}\right| \Psi_{n}\right\rangle\left\langle\Psi_{n}\left|\mu^{x}\right| \Psi_{f}\right\rangle}{\Omega_{n g}+\omega_{2 y}-i \epsilon}\right) \\
& =-\left(\left\langle\Psi_{g}\left|\mu^{x}\right| X_{f}^{-\epsilon, \omega_{2 y}}\right\rangle+\left\langle\tilde{X}_{g}^{-\epsilon, \omega_{2 y}}\left|\mu^{x}\right| \Psi_{f}\right\rangle\right)
\end{aligned}
$$

where the $\left|X_{k}^{\epsilon, \omega_{x}}\right\rangle$ and $\left\langle\tilde{X}_{k}^{\epsilon, \omega_{x}}\right|$ are the complex right and left first-order response wave functions of state $k$ due to the perturbing electric field of frequency $\omega_{1 x}$ polarized along the $x$ direction. These response wave functions depend parametrically on $\epsilon$ and are given, according to first-order perturbation theory, as ${ }^{16,21,28}$

$$
\left|X_{k}^{\epsilon, \omega_{1 x}}\right\rangle=\sum_{n}\left|\Psi_{n}\right\rangle \frac{\left\langle\Psi_{n}\left|\mu^{x}\right| \Psi_{k}\right\rangle}{\Omega_{n k}-\omega_{1 x}-i \epsilon}
$$

and

$$
\left\langle\tilde{X}_{k}^{\epsilon, \omega_{1 x}}\right|=\sum_{n} \frac{\left\langle\Psi_{n}\left|\mu^{x}\right| \Psi_{k}\right\rangle}{\Omega_{n k}+\omega_{1 x}+i \epsilon}\left\langle\Psi_{n}\right| .
$$


These first-order many-body response functions are computed iteratively by solving the response equations

$$
\left(H-E_{k}-\omega_{1 x}-i \epsilon\right) X_{k}^{\epsilon, \omega_{1 x}}=\left\langle\Phi_{\nu}\left|\mu^{x}\right| \Psi_{k}\right\rangle
$$

and

$$
\tilde{X}_{k}^{\epsilon, \omega_{1 x}}\left(H-E_{k}+\omega_{1 x}+i \epsilon\right)=\left\langle\Psi_{k}\left|\mu^{x}\right| \Phi_{\nu}\right\rangle,
$$

where $\left\{\Phi_{\nu}\right\}$ is the set of $\nu$-tuply excited Slater determinants from the target-states manifold; e.g., in EOM-CCSD damped response theory, $\left\{\Phi_{\nu}\right\}$ spans the reference, singly excited, and doubly excited determinants. We recast Eqs. (5) and (6) as

$$
M_{g \rightarrow f}^{x y}\left(\omega_{1 x},+\epsilon\right)=\sum_{p q} \gamma_{p q}^{\epsilon, x} \mu_{p q}^{y}
$$

and

$$
M_{f \rightarrow g}^{x y}\left(\omega_{2 y},-\epsilon\right)=\sum_{p q} \tilde{\gamma}_{p q}^{-\epsilon, y} \mu_{p q}^{x},
$$

where $\gamma^{x}$ and $\tilde{\gamma}^{y}$ are the complex response reduced 1PTDMs given by

$$
\gamma_{p q}^{\epsilon, x}=\gamma_{p q}^{+\epsilon, \omega_{1 x}}=-\left(\left\langle\Psi_{f}\left|\hat{p}^{\dagger} \hat{q}\right| X_{g}^{\epsilon, \omega_{1 x}}\right\rangle+\left\langle\tilde{X}_{f}^{\epsilon, \omega_{1 x}}\left|\hat{p}^{\dagger} \hat{q}\right| \Psi_{g}\right\rangle\right)
$$

and

$$
\tilde{\gamma}_{p q}^{-\epsilon, y}=\tilde{\gamma}_{p q}^{-\epsilon, \omega_{2 y}}=-\left(\left\langle\Psi_{g}\left|\hat{p}^{\dagger} \hat{q}\right| X_{f}^{-\epsilon, \omega_{2 y}}\right\rangle+\left\langle\tilde{X}_{g}^{-\epsilon, \omega_{2 y}}\left|\hat{p}^{\dagger} \hat{q}\right| \Psi_{f}\right\rangle\right),
$$

where $\hat{p}^{\dagger}$ and $\hat{q}$ are the creation and annihilation operators in molecular orbitals $\phi_{p}$ and $\phi_{q}$, respectively. Following our previous $\operatorname{work}^{16}$, we use $\omega \mathrm{DM}$ to denote the individual components of 1PTDMs on the RHS of Eqs. (13) and (14) between a frequency-dependent response state and a zero-order state. Thus, $\gamma^{\epsilon, x}$ is the sum of a $\omega$ DM between the final and response ground state and another $\omega \mathrm{DM}$ between the initial and response final state.

For one-photon transitions, the reduced 1PTDM can be interpreted as the exciton's wave function according to

$$
\Psi_{e x c}\left(r_{h}, r_{p}\right)=\sum_{p q} \gamma_{p q} \phi\left(r_{h}\right) \phi\left(r_{p}\right),
$$

where $r_{h}$ and $r_{p}$ are the hole and electron (particle) coordinates ${ }^{12,14,32}$. For second-order (two-photon) RIXS process, the exciton's wave function (as well as response 1PTDMs) has polarized components along the three Cartesian components $(\hat{x}, \hat{y}$, and $\hat{z})$ :

$$
\Psi_{e x c}^{\epsilon, x}\left(r_{h}, r_{p}\right)=\sum_{p q} \gamma_{p q}^{\epsilon, x} \phi_{q}\left(r_{h}\right) \phi_{p}\left(r_{p}\right)
$$


and

$$
\Psi_{e x c}^{\epsilon}\left(r_{h}, r_{p}\right)=\Psi_{e x c}^{\epsilon, x}\left(r_{h}, r_{p}\right) \hat{x}+\Psi_{e x c}^{\epsilon, y}\left(r_{h}, r_{p}\right) \hat{y}+\Psi_{e x c}^{\epsilon, z}\left(r_{h}, r_{p}\right) \hat{z}
$$

In spatial representation, the exciton's wave function provides a visual map of how the electronic distribution changes upon the transition ${ }^{12,14,33,34}$. It can also be used to compute various physical quantities, such as the correlation between the hole and electron and their average separation ${ }^{12,15}$ :

$$
\begin{aligned}
d_{e x c} & =\sqrt{\left\langle\Psi_{e x c}\left(r_{h}, r_{p}\right)\left|\left(r_{h}-r_{p}\right)^{2}\right| \Psi_{e x c}\left(r_{h}, r_{p}\right)\right\rangle} \\
& =\sqrt{\frac{\left\|\gamma^{\epsilon, x}\right\|^{2}\left(d_{e x c}^{\epsilon, x}\right)^{2}+\left\|\gamma^{\epsilon, y}\right\|^{2}\left(d_{e x c}^{\epsilon, y}\right)^{2}+\left\|\gamma^{\epsilon, z}\right\|^{2}\left(d_{e x c}^{\epsilon, z}\right)^{2}}{\left\|\gamma^{\epsilon, x}\right\|^{2}+\left\|\gamma^{\epsilon, y}\right\|^{2}+\left\|\gamma^{\epsilon, z}\right\|^{2}}}
\end{aligned}
$$

where

$$
\begin{aligned}
& d_{e x c}^{\epsilon, x}=\sqrt{\left\langle\Psi_{e x c}^{\epsilon, x}\left(r_{h}, r_{p}\right)\left|\left(r_{h}-r_{p}\right)^{2}\right| \Psi_{e x c}^{\epsilon, x}\left(r_{h}, r_{p}\right)\right\rangle} \\
& =\sqrt{\frac{\left\|\gamma^{\epsilon, x, \operatorname{Re}}\right\|\left\|^{2}\left(d_{e x c}^{\epsilon, x, \operatorname{Re}}\right)^{2}+\right\| \gamma^{\epsilon, x, \operatorname{Im}} \|^{2}\left(d_{e x c}^{\epsilon, x, \operatorname{Im}}\right)^{2}}{\left\|\gamma^{\epsilon, x, \operatorname{Re}}\right\|^{2}+\left\|\gamma^{\epsilon, x, \operatorname{Im}}\right\|^{2}}}, \\
& d_{e x c}^{\epsilon, x, \operatorname{Re} / \operatorname{Im}}=\sqrt{\left\langle\Psi_{\text {exc }}^{\epsilon, x, \operatorname{Re} / \operatorname{Im}}\left(r_{h}, r_{p}\right)\left|\left(r_{h}-r_{p}\right)^{2}\right| \Psi_{\text {exc }}^{\epsilon, x, \operatorname{Re} / \mathrm{Im}}\left(r_{h}, r_{p}\right)\right\rangle},
\end{aligned}
$$

and

$$
\left\|\gamma^{\epsilon, x}\right\|^{2}=\left\|\gamma^{\epsilon, x, \operatorname{Re}}\right\|^{2}+\left\|\gamma^{\epsilon, x, \operatorname{Im}}\right\|^{2} .
$$

These exciton descriptors facilitate the assignment of the transitions in terms of valence, Rydberg, or charge-transfer character ${ }^{12,15}$.

The description of exciton's wave function for a one-photon transition is the most concise in terms of NTOs, which are computed by means of unitary orbital transformations ${ }^{12,13,16}$. This is achieved by singular value decomposition (SVD) of the 1PTDM as follows:

$$
\gamma=\mathbf{V} \mathbf{\Sigma} \mathbf{U}^{\mathbf{T}}
$$

where $\boldsymbol{\Sigma}$ is the diagonal matrix of singular values, $\sigma_{K} \mathrm{~s}$, and matrices $\mathbf{V}$ and $\mathbf{U}$ contain the hole and particle NTOs according to

$$
\psi_{K}^{p}(r)=\sum_{q} V_{q K} \phi_{q}(r)
$$

and

$$
\psi_{K}^{h}(r)=\sum_{q} U_{q K} \phi_{q}(r)
$$


The squares of $\sigma_{K} \mathrm{~s}$ can be interpreted as the weights of the respective NTO pair when divided by the square of the Frobenius norm of $\gamma$ :

$$
\|\gamma\|^{2} \equiv \sum_{p q} \gamma_{p q}^{2}=\sum_{k} \sigma_{K}^{2}
$$

In the analyses below, we report such normalized singular values:

$$
\sigma_{K}^{\prime}=\frac{\sigma_{K}}{\|\gamma\|}
$$

which is equivalent to using normalized 1PTDMs in the wave-function analysis.

The SVD procedure removes the arbitrariness associated with the orbital choice. Since only a handful of $\sigma_{K}$ s are non-negligible, the NTO representation enables the most compact molecular orbital representation of any transition, including the transitions between multiconfigurational correlated wave functions. In terms of NTOs, the exciton's wave function for a one-photon transition is given by

$$
\Psi_{\text {exc }}\left(r_{h}, r_{p}\right)=\sum_{K} \sigma_{K} \psi_{K}^{p}\left(r_{p}\right) \psi_{K}^{h}\left(r_{h}\right)
$$

In contrast to the real-valued exciton's wave functions and 1PTDMs for one-photon and two-photon absorption processes in Refs. 12, 13, and 16, the exciton's wave function and response 1PTDMs for the RIXS process are complex because within the damped response theory formalism the response wave functions become complex. Below we explain how to interpret these complex 1TPDMs.

Rewriting the response 1PTDM in Eq. (16), we obtain

$$
\Psi_{e x c}^{\epsilon, x}\left(r_{h}, r_{p}\right)=\sum_{p q} \gamma_{p q}^{\epsilon, x, \operatorname{Re}} \phi_{q}\left(r_{h}\right) \phi_{p}\left(r_{p}\right)+i \sum_{p q} \gamma_{p q}^{\epsilon, x, \operatorname{Im}} \phi_{q}\left(r_{h}\right) \phi_{p}\left(r_{p}\right)
$$

Since the real and imaginary components of the RIXS scattering moments respectively accumulate the off-resonance and near-resonance SOS terms, the corresponding real and imaginary response 1PTDMs respectively provide the cumulative orbital information of these off-resonance and near-resonance terms. We reformulate Eq. (28) to

$$
\begin{aligned}
\Psi_{e x c}^{\epsilon, x}\left(r_{h}, r_{p}\right) & =\sum_{K} \sigma_{K}^{\epsilon, x, \operatorname{Re}} \psi_{K}^{\epsilon, x, \operatorname{Re}}\left(r_{p}\right) \psi_{K}^{\epsilon, x, \operatorname{Re}}\left(r_{h}\right) \\
& +i \sum_{L} \sigma_{L}^{\epsilon, x, \operatorname{Im}} \psi_{L}^{\epsilon, x, \operatorname{Im}}\left(r_{p}\right) \psi_{L}^{\epsilon, x, \operatorname{Im}}\left(r_{h}\right)
\end{aligned}
$$


by performing SVD on the real and imaginary response 1PTDMs separately, so that two sets of real NTOs $\left(\left\{\psi_{K}^{\mathrm{Re}}\left(r_{p}\right), \psi_{K}^{\mathrm{Re}}\left(r_{h}\right)\right\}\right.$ and $\left.\left\{\psi_{L}^{\operatorname{Im}}\left(r_{p}\right), \psi_{L}^{\operatorname{Im}}\left(r_{h}\right)\right\}\right)$ are obtained and used for visualization and interpretation.

The relative significance of the off-resonance and near-resonance terms for a given RIXS moment can be estimated from the norms of the real and imaginary response 1PTDMs, $\left\|\gamma^{\epsilon, x, \operatorname{Re}}\right\|$ and $\left\|\gamma^{\epsilon, x, \operatorname{Im}}\right\|$. For example, if $v^{x, \operatorname{Im}}=\frac{\left\|\gamma^{\epsilon, x, \operatorname{Im}}\right\|^{2}}{\left\|\gamma^{\epsilon, x}\right\|^{2}} \approx 1$ or $v^{x, \operatorname{Re}}=\frac{\left\|\gamma^{\epsilon, x, \operatorname{Re}}\right\|^{2}}{\left\|\gamma^{\epsilon, x}\right\|^{2}} \approx 0$, the corresponding $x$ component of the exciton's wave function can be approximated by just the imaginary near-resonance contributions. Similarly, the relative significance of the offresonance and near-resonance terms for the overall RIXS transition can be estimated using the norms of response 1PTDMs along the three Cartesian coordintates. For example, if $\Upsilon^{\operatorname{Im}}=\Upsilon^{x, \operatorname{Im}}+\Upsilon^{y, \operatorname{Im}}+\Upsilon^{z, \operatorname{Im}} \approx 1$ or $\Upsilon^{\operatorname{Re}}=\Upsilon^{x, \operatorname{Re}}+\Upsilon^{y, \operatorname{Re}}+\Upsilon^{z, \operatorname{Re}} \approx 0$, where $\Upsilon^{x, \operatorname{Im}}=\frac{\left\|\gamma^{\epsilon, x, \operatorname{Im}}\right\|^{2}}{\sum_{x, y, z}\left\|\gamma^{\epsilon, x}\right\|^{2}}$ and $\Upsilon^{x, \operatorname{Re}}=\frac{\left\|\gamma^{\epsilon, x, \operatorname{Re}}\right\|^{2}}{\sum_{x, y, z}\left\|\gamma^{\epsilon, x}\right\|^{2}}$, then the RIXS transition has predominant contributions from nearresonance channels. In the discussion that follows, we drop the index $\epsilon$ in $\gamma^{\epsilon, x, \operatorname{Re}}$ and $\gamma^{\epsilon, x, \operatorname{Im}}$ for brevity.

\section{COMPUTATIONAL DETAILS}

Using the existing infrastructure of the libwfa library ${ }^{15}$ for wave-function analysis ${ }^{12,13}$, we implemented the calculations of the NTOs for RIXS 1PTDMs in a development version of QChem ${ }^{35,36}$. Below we illustrate the utility of this orbital analysis for the RIXS transitions in the $\mathrm{OH}$ radical, benzene, para-nitroaniline, and 4-amino-4'-nitrostilbene. In all calculations, we employ the recently developed implementation ${ }^{21}$ of RIXS calculations within the fcCVS-EOM-EE-CCSD framework ${ }^{37}$. For the OH radical, benzene, and pNA, we use the $6-311(2+,+) \mathrm{G}^{* *}$ basis set with the uncontracted core $(\mathrm{uC})$ functions ${ }^{38}$. For the OH radical, we use the experimental bond length of $0.9697 \AA$. We use the geometries from Refs. 21 and 16 for benzene and pNA, respectively. For 4A4NS, we use the B3LYP/6-311G** optimized geometry and the $6-31+\mathrm{G}^{*}$ basis set with the uncontracted core functions for XAS, XES, and RIXS calculations. Relevant Cartesian coordinates are provided in the Supplemental Materials (SI). The phenomenological damping parameter $\epsilon$ was set to $0.005(\mathrm{OH}), 0.01$ (benzene and pNA), and $0.03(4 \mathrm{~A} 4 \mathrm{NS})$.

We use Q-Chem's symmetry notations throughout this letter (more details can be found in Refs. 21 and 16 and at http://iopenshell.usc.edu/resources/howto/symmetry/). We use the 
$C_{2 v}$ symmetry group for $\mathrm{OH}$ and $\mathrm{pNA}, D_{2 h}$ symmetry group for benzene, and $C_{s}$ symmetry group for 4A4NS. We visualize the NTOs and canonical MOs with Gabedit ${ }^{39}$ and IQMol ${ }^{40}$, respectively.

\section{RESULTS AND DISCUSSION}

\section{A. Wave-function analysis of RIXS transition in $\mathrm{OH}$}

In the $\mathrm{OH}$ radical, the $\pi_{y}$ orbital is singly occupied (see Fig. 3) and the lowest resonant xray absorption involves the excitation of the $1 s_{O}$ electron to fill the valence $\pi$ hole $^{41}$. This is clearly shown by the NTO analysis of the $\mathrm{XB}_{2} \rightarrow \mathrm{c} 1 \mathrm{~A}_{1}$ transition ("c" denotes a core-excited state), which is given in Fig. 4 (and the SI).

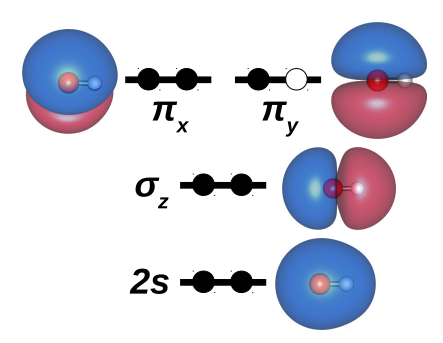

15

FIG. 3: Molecular orbitals and ground-state electronic configuration of the $\mathrm{OH}$ radical. Electronic states involving excitation of $1 s_{O}$ electrons are denoted by prefix 'c'.

The lowest valence excited state is the $1 \mathrm{~A}_{1}$ state at $4.15 \mathrm{eV}$. Within the three-states model, the $\mathrm{XB}_{2} \rightarrow 1 \mathrm{~A}_{1}$ RIXS transition with the incoming photon's energy tuned at the $\mathrm{XB}_{2} \rightarrow \mathrm{c} \mathrm{A}_{1}$ resonance entails $\mathrm{x}$-ray absorption from the ground state to the $\mathrm{c}_{1} \mathrm{~A}_{1}$ state and $\mathrm{x}$-ray emission from the $\mathrm{c}_{1} \mathrm{~A}_{1}$ state to the final valence $1 \mathrm{~A}_{1}$ state. This transition is the dominant inelastic feature in the RIXS spectrum of aqueous $\mathrm{OH}^{8}$. The NTO analysis for the $\mathrm{c}^{\mathrm{A}} \mathrm{A}_{1} \rightarrow 1 \mathrm{~A}_{1}$ emission is shown in Fig. 4, which indicates $\sigma_{z} \rightarrow 1 s$ character. Based on this three-states model, one can now identify the $\sigma_{z} \rightarrow 1 s \rightarrow \pi_{y}$ orbital channel as the dominant pathway in the $\mathrm{XB}_{2} \rightarrow 1 \mathrm{~A}_{1}$ RIXS transition.

Let us now compare this approximate analysis based on the three-states model with the NTO analysis of the RIXS 1PTDMs. In the analytic RIXS calculations, we find that the 


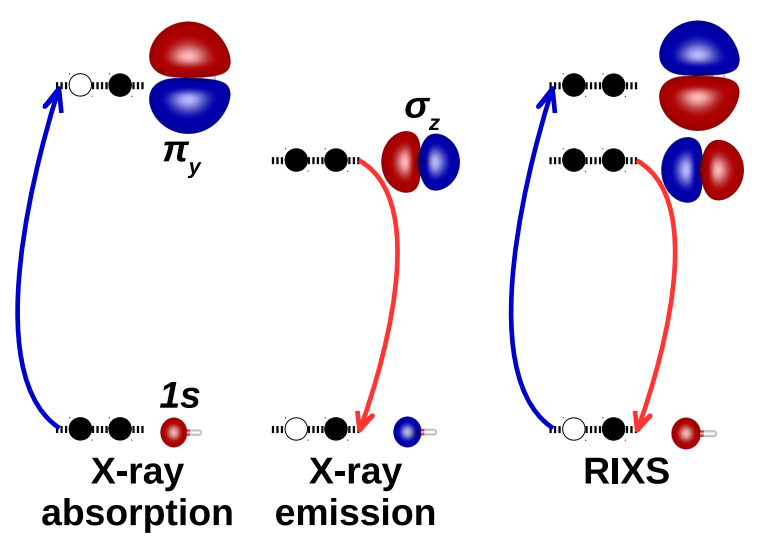

FIG. 4: NTO analysis of the $\mathrm{XB}_{2} \rightarrow 1 \mathrm{~A}_{1}$ RIXS transition in the $\mathrm{OH}$ radical. Comparison of important NTO pairs computed separately for the x-ray absorption $\left(\mathrm{XB}_{2} \rightarrow \mathrm{c} 1 \mathrm{~A}_{1}\right)$ and $\mathrm{x}$-ray emission $\left(\mathrm{c}_{1} \mathrm{~A}_{1} \rightarrow 1 \mathrm{~A}_{1}\right)$ transitions with the NTO pairs computed from the RIXS 1PTDMs show that the three-states model is appropriate for this transition.

imaginary $M^{y z}$ components - and thus the near-resonance orbital channels - are dominant for the $\mathrm{XB}_{2} \rightarrow 1 \mathrm{~A}_{1}$ RIXS transition and, therefore, have the dominant contribution to the cross section. The RIXS 1PTDMs corresponding to the imaginary $M_{g \rightarrow f}^{y z}$ and $M_{f \rightarrow g}^{y z}$ components are the $\gamma^{y, \operatorname{Im}}$ and $\tilde{\gamma}^{z, \mathrm{Im}} 1 \mathrm{PTDMs}$, respectively. The detailed NTO analyses of these RIXS 1PTDMs are given in the SI.

By inspecting the norms of the imaginary RIXS 1PTDMs and of the respective $\omega$ DMs we note that only the first terms in Eqs. (13) and (14) provide significant contribution. Thus, the NTOs of the full imaginary RIXS 1PTDMs can be explained by interpreting these imaginary $\omega$ DMs. The first imaginary $\omega \mathrm{DM}$ in Eq. (13) reflects the transition from the "virtual" $X_{g}$ state (first-order response ground state) to the final state and so the NTO pairs correspond to the transition that fills the core hole (emission). Complementary to this orbital transition, the NTO pairs from the first imaginary $\omega$ DM in Eq. (14) reflect the transition from the $X_{f}$ "virtual" state (first-order perturbed final state) to the initial state, i.e., the reverse of core-hole formation (reverse of absorption). By joining these two sets of NTO pairs together, the orbital picture of the RIXS transition is constructed. For the $\mathrm{XB}_{2} \rightarrow 1 \mathrm{~A}_{1}$ RIXS transition, each set consists of one dominant NTO pair (see the SI). The analysis of $\gamma^{y, I m}$ identifies the $\sigma_{z}$ hole and $1 s$ particle NTOs; the analysis of $\tilde{\gamma}^{z, \mathrm{Im}}$ identifies the $\pi_{y}$ hole and $1 s$ particle NTOs. Using the norms of response 1PTDMs, we get $\Upsilon^{\operatorname{Im}}$ equal to 1.00 for this transition. Thus, the dominant RIXS channel is resonant and 
given by $\sigma_{z} \rightarrow 1 s \rightarrow \pi_{y}$ with its $1 s \rightarrow \pi_{y}$ excitation and $\sigma_{z} \rightarrow 1 s$ de-excitation components. This is consistent with the three-states model described above, indicating that for this RIXS transition the three-states model provides a good approximation to the full SOS expression.

\section{B. Wave-function analysis of RIXS transitions in benzene}

Fig. 5 shows occupied molecular orbitals of benzene. The six $1 s_{C}$ core orbitals form six nearly degenerate delocalized molecular orbitals. Depending on the symmetry of the target orbital, different core orbitals are active in the XAS transitions ${ }^{21}$. The relevant

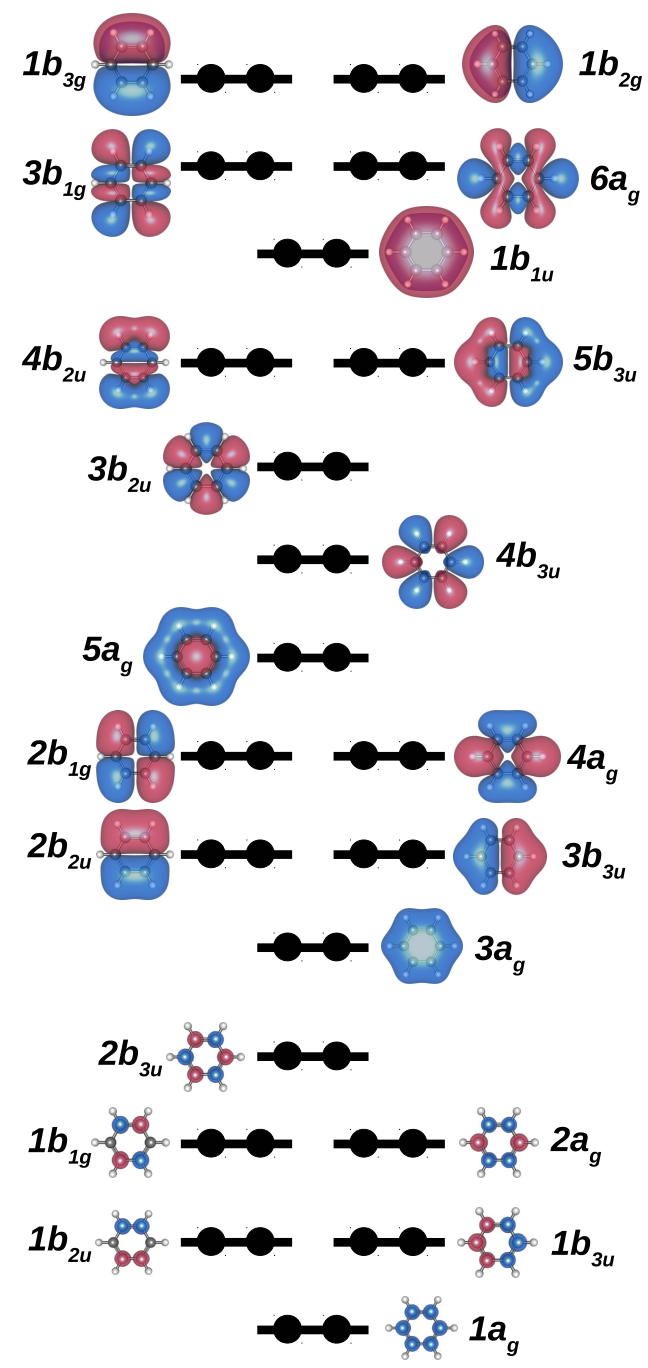

FIG. 5: Molecular orbitals and ground-state electronic configuration of benzene. The six core orbitals and the electronic states involving excitations from these orbitals are denoted by prefix 'c'. 
virtual molecular orbitals (not shown) are doubly degenerate $\pi^{*}$ LUMO and diffuse $s$ and $p$ Rydberg orbitals.

The two dominant features in the XAS spectrum of benzene ${ }^{42-49}$ are peak A and peak B at $285.97 \mathrm{eV}$ and $287.80 \mathrm{eV}$, respectively (theoretical values ${ }^{21}$ computed with fc-CVS-EOM-EECCSD/uC-6-311 $\left.(2+,+) \mathrm{G}^{* *}\right)$. When the incoming photon's energy is tuned to the peak-A resonance, the dominant inelastic feature is the energy-loss peak at $10.67 \mathrm{eV}$, characterized by equal contributions from the degenerate $\mathrm{XA}_{g} \rightarrow 13 \mathrm{~B}_{2 g}$ and $\mathrm{XA}_{g} \rightarrow 12 \mathrm{~B}_{3 g}$ transitions. In contrast, when the incoming photon's energy is tuned to the peak-B resonance, the dominant inelastic feature is the energy-loss peak at $6.45 \mathrm{eV}$, characterized by equal contributions from the degenerate $\mathrm{XA}_{g} \rightarrow 1 \mathrm{~B}_{2 g}$ and $\mathrm{XA}_{g} \rightarrow 1 \mathrm{~B}_{3 g}$ transitions. Below we show the NTO analysis of only the $\mathrm{XA}_{g} \rightarrow 13 \mathrm{~B}_{2 g}$ and $\mathrm{XA}_{g} \rightarrow 1 \mathrm{~B}_{2 g}$ RIXS transitions with incoming photon energies tuned at peak-A and peak-B resonances, respectively. The NTO analyses for the two transitions are similar except for the differences in symmetry labels of the orbitals.

The NTO analysis for the dark one-photon $\mathrm{XA}_{g} \rightarrow 13 \mathrm{~B}_{2 g}$ transition (given in the SI) suggests that this valence transition is made up of two orbital transitions: $b_{2 u} \rightarrow a_{u}$ and $b_{3 u} \rightarrow b_{1 u}$. Similarly, the NTO analysis of XAS peak $\mathrm{A}\left(\mathrm{XA}_{g} \rightarrow c 2 \mathrm{~B}_{1 u}\right)$ transition in Fig. 6 shows two dominant orbital transitions: $c b_{1 g} \rightarrow a_{u}$ and $c a_{g} \rightarrow b_{1 u}$. Similarly, the

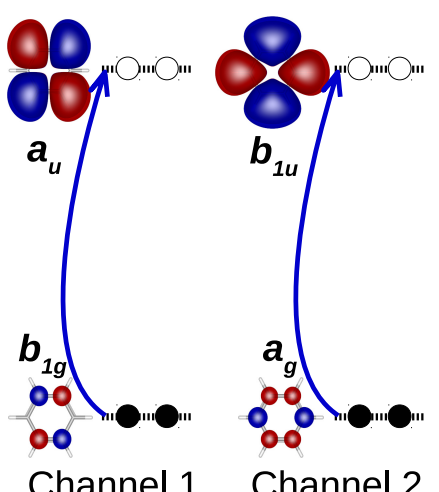

X-ray absorption

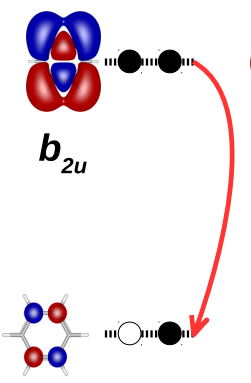

Channel 1

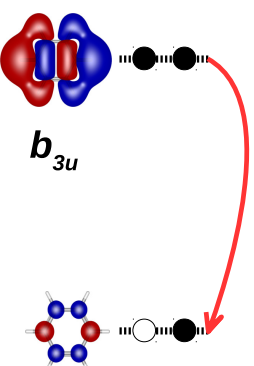

Channel 2

$\mathrm{X}$-ray emission

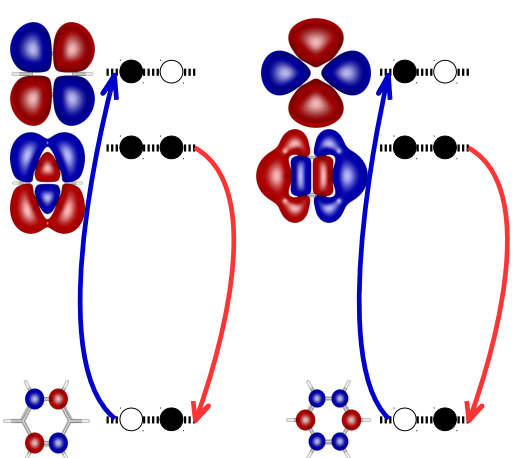

Channel 1

RIXS

FIG. 6: NTO analysis of the $\mathrm{XA}_{g} \rightarrow 13 \mathrm{~B}_{2 g}$ RIXS transition in benzene. Comparison of important NTO pairs computed separately for the x-ray absorption $\left(\mathrm{XA}_{g} \rightarrow \mathrm{c} 2 \mathrm{~B}_{1 u}\right)$ and $\mathrm{x}$-ray emission $\left(\mathrm{c}_{2} \mathrm{~B}_{1 u} \rightarrow 13 \mathrm{~B}_{2 g}\right)$ transitions with the NTO pairs computed from the RIXS 1PTDMs shows that the three-states model is adequate for this RIXS transition. Both orbital channels contribute significantly into this transition. 
NTO analysis of the $\mathrm{c}^{2} \mathrm{~B}_{1 u} \rightarrow 13 \mathrm{~B}_{2 g}$ x-ray emission shows two dominant orbital transitions: $b_{2 u} \rightarrow c b_{1 g}$ and $b_{3 u} \rightarrow c a_{g}$. Based on these analyses, the three-states model for the $\mathrm{XA}_{g} \rightarrow$ $13 \mathrm{~B}_{2 g}$ RIXS transition identifies two important orbital channels: $b_{2 u} \rightarrow c b_{1 g} \rightarrow a_{u}$ and $b_{3 u} \rightarrow c a_{g} \rightarrow b_{1 u}$.

The $g \rightarrow f$ and $f \rightarrow g$ RIXS moment tensors are dominated by the imaginary $z x$ components. This is also reflected in the norms of the imaginary 1PTDMs given in the SI, which are a few orders of magnitude larger than those of real 1 PTDMs $\left(\Upsilon^{\operatorname{Im}}=1.00\right)$. The NTO analyses of the $\gamma^{z, \operatorname{Im}}$ and $\tilde{\gamma}^{x, \operatorname{Im}}$ RIXS 1PTDMs of the $\mathrm{XA}_{g} \rightarrow 13 \mathrm{~B}_{2 g}$ shown in Fig. 6 and in the SI identifies two dominant near-resonance orbital channels: $b_{2 u} \rightarrow c b_{1 g} \rightarrow a_{u}$ and $b_{3 u} \rightarrow c a_{g} \rightarrow b_{1 u}$. In other words, $c b_{1 g}$ and $c a_{g}$ are the intermediate core orbitals that facilitate the two-photon inelastic scattering, driving the electronic density from the $b_{2 u}$ orbital to the $2 a_{u}$ orbital and from the $b_{3 u}$ orbital to the $4 b_{1 u}$ orbital, respectively. This orbital analysis of the RIXS 1PTDMs is consistent with the approximate analysis from the three-states model.

The one-photon $\mathrm{XA}_{g} \rightarrow 1 \mathrm{~B}_{2 g}$ transition is dark; its NTO analysis given in the SI reveals its dominant HOMO-LUMO character $\left(b_{2 g} \rightarrow a_{g}\right)$ and a miniscule contribution from the $b_{1 u} \rightarrow b_{3 u}$ transition. The NTO analysis of XAS peak B transition $\left(\mathrm{XA}_{g} \rightarrow \mathrm{c} 2 \mathrm{~B}_{3 u}\right)$ shown

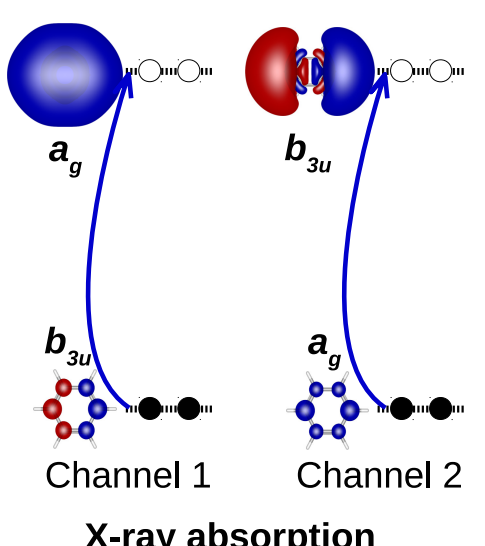

X-ray absorption

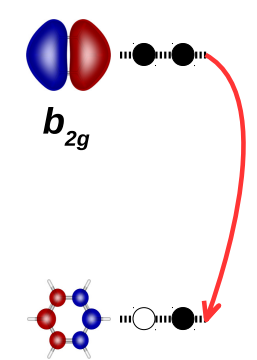

Channel 1

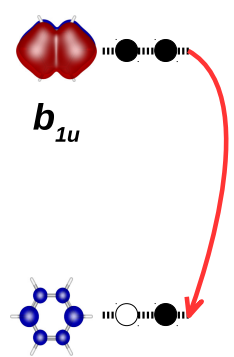

Channel 2

$X$-ray emission

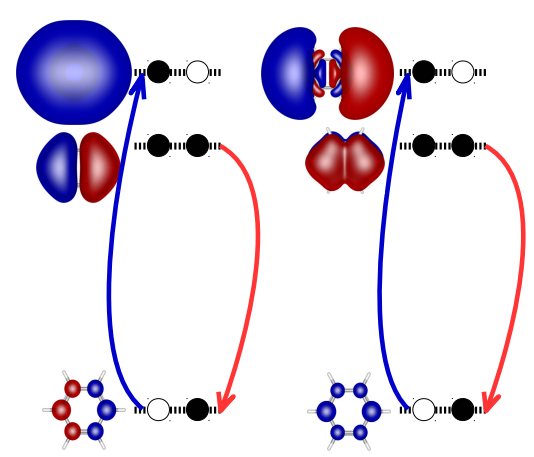

Channel 1

\section{RIXS}

FIG. 7: NTO analysis of the $1 \mathrm{~A}_{g} \rightarrow 1 \mathrm{~B}_{2 g}$ RIXS transition in benzene. Comparison of important NTO pairs computed separately for the x-ray absorption $\left(1 \mathrm{~A}_{g} \rightarrow \mathrm{c} 2 \mathrm{~B}_{3 u}\right)$ and $\mathrm{x}$-ray emission $\left(\mathrm{c}^{2} \mathrm{~B}_{3 u} \rightarrow 1 \mathrm{~B}_{2 g}\right)$ transitions with the NTO pairs computed from the RIXS 1PTDMs shows that the three-states model is adequate for this RIXS transition. Orbital channel 1 provides dominant contributions. 
in the SI and Fig. 7 indicates that this core excitation has predominantly $c b_{3 u} \rightarrow a_{g}$ orbital character, with a small contribution from the $c a_{g} \rightarrow b_{3 u}$ transition. The NTO analysis of the $c 2 \mathrm{~B}_{3 u} \rightarrow 1 \mathrm{~B}_{2 g}$ X-ray emission transition has a predominantly $b_{2 g} \rightarrow c b_{3 u}$ character with a small contribution from the $b_{1 u} \rightarrow c a_{g}$ transition. Thus, within the three-states model, the NTO analyses of the $\mathrm{XA}_{g} \rightarrow \mathrm{c} 2 \mathrm{~B}_{3 u} \mathrm{x}$-ray absorption and $\mathrm{c} 2 \mathrm{~B}_{3 u} \rightarrow 1 \mathrm{~B}_{2 g}$ x-ray emission identify the orbital character as $b_{2 g} \rightarrow c b_{3 u} \rightarrow a_{g}$, with a small contribution from the $b_{1 u} \rightarrow c a_{g} \rightarrow b_{3 u}$ channel.

The RIXS moment tensor for the $\mathrm{XA}_{g} \rightarrow 1 \mathrm{~B}_{2 g}$ transition is dominated by the imaginary component of $M^{x z}$ moments. Here, we perform NTO analyses of the $\gamma^{x, \operatorname{Im}}$ and $\tilde{\gamma}^{z, \operatorname{Im}} \operatorname{RIXS}$ 1PTDMs corresponding to the $M_{g \rightarrow f}^{x z}$ and $M_{f \rightarrow g}^{x z}$ components, respectively. The analysis of $\gamma^{x, I m}$ identifies the $b_{2 g} \rightarrow c b_{3 u}$ NTO pair as dominant, with miniscule contribution from the $b_{1 u} \rightarrow c a_{g}$ NTO pair. The analysis of $\tilde{\gamma}^{z, \text { Im }}$ identifies the dominant $a_{g} \rightarrow c b_{3 u}$ NTO pair and a less important $b_{3 u} \rightarrow c a_{g}$ NTO pair. Combining these two analyses, the dominant orbital channel is $b_{2 g} \rightarrow c b_{3 u} \rightarrow a_{g}$. We get $\Upsilon^{\operatorname{Im}}$ equal to 0.98 for this RIXS transition. This is consistent with the analysis from the three-states model discussed above. Similarly, the dominant RIXS channel is resonant and given by $b_{3 g} \rightarrow c b_{2 u} \rightarrow a_{g}$ for the $\mathrm{XA}_{g} \rightarrow 1 \mathrm{~B}_{3 g}$ transition.

\section{Wave-function analysis of RIXS transitions in para-nitroaniline}

The orbital analysis of the selected RIXS transitions in the $\mathrm{OH}$ radical and benzene supports the notion that the dominant orbital channel in RIXS is (nearly) resonant and that three-states models are sufficient for determining the important orbitals involved in the RIXS transition. In this section, we present a counter example illustrating the limitations of few-core-excited-states models. We consider RIXS transitions in para-nitroaniline (pNA) and show that for this system the predominant channel driving the electronic density in the course of inelastic scattering may or may not be (nearly) resonant in character.

Fig. 8 shows occupied molecular orbitals of pNA. The special feature of this molecule is that the lowest excited state has strong intermolecular charge-transfer character ${ }^{50-53}$. This is the lowest fully symmetric $\mathrm{XA}_{1} \rightarrow 2 \mathrm{~A}_{1}$ transition with large oscillator strength $(f=$ 0.4). The NTO analysis, provided in the SI, shows that this transition can be described as HOMO-LUMO excitation: $\pi\left(b_{2}\right) \rightarrow \pi^{*}\left(b_{2}\right)$. 


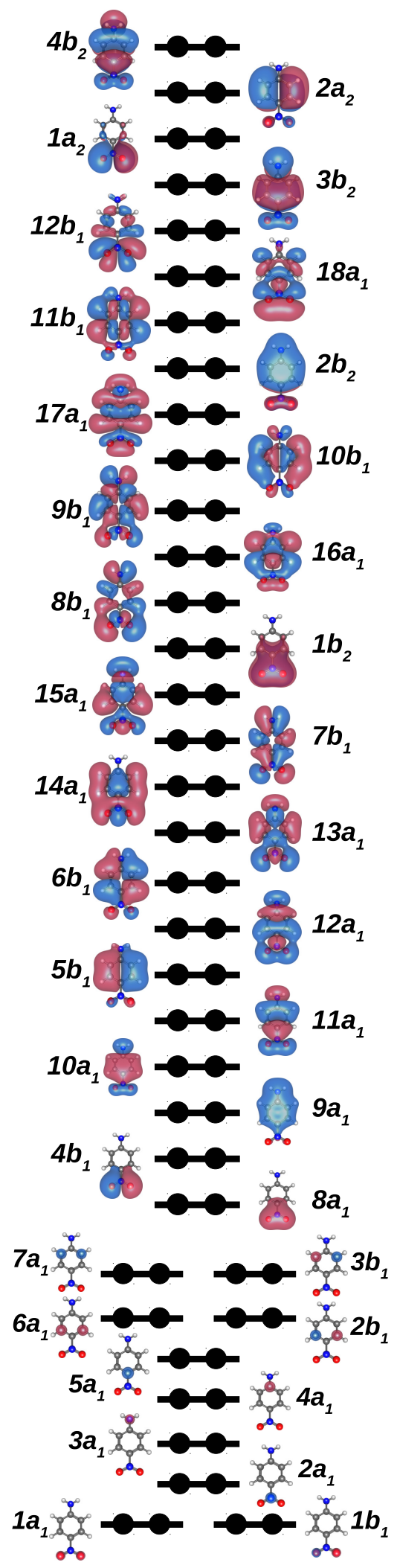

FIG. 8: Occupied molecular orbital diagram for para-nitroaniline. Core orbitals and the electronic states involving excitations from these orbitals are denoted by prefix 'c'. 
The strong charge-transfer character of this transition $(\Delta \mu=3.2$ a.u. $)$ has non-trivial consequences on the character of the 2PA transition, as was discussed in Ref. 16. Specifically, we have shown that the $2 \mathrm{PA}$ moments for the $\mathrm{XA}_{1} \rightarrow 2 \mathrm{~A}_{1}$ transition in pNA can be described by the two-states model involving just the initial and final states, in contrast to other $2 \mathrm{PA}$ examples involving specific virtual states giving dominant contributions to the cross sections. Thus, for this transition, the 2PA transition moments are given according to

$$
\begin{aligned}
M_{g \rightarrow f}^{x y} \approx & -\sum_{n=g, f}\left(\frac{\left\langle\Psi_{f}\left|\mu^{y}\right| \Psi_{n}\right\rangle\left\langle\Psi_{n}\left|\mu^{x}\right| \Psi_{g}\right\rangle}{\Omega_{n g}-\omega_{1 x}-i \epsilon}+\frac{\left\langle\Psi_{f}\left|\mu^{x}\right| \Psi_{n}\right\rangle\left\langle\Psi_{n}\left|\mu^{y}\right| \Psi_{g}\right\rangle}{\Omega_{n g}-\omega_{2 y}-i \epsilon}\right) \\
\approx & -\left\langle\Psi_{f}\left|\mu^{y}\right| \Psi_{g}\right\rangle \frac{\left\langle\Psi_{f}\left|\mu^{x}\right| \Psi_{f}\right\rangle-\left\langle\Psi_{g}\left|\mu^{x}\right| \Psi_{g}\right\rangle}{\omega_{1 x}+i \epsilon} \\
& -\left\langle\Psi_{f}\left|\mu^{x}\right| \Psi_{g}\right\rangle \frac{\left\langle\Psi_{f}\left|\mu^{y}\right| \Psi_{f}\right\rangle-\left\langle\Psi_{g}\left|\mu^{y}\right| \Psi_{g}\right\rangle}{\omega_{2 y}+i \epsilon}
\end{aligned}
$$

and

$$
\begin{aligned}
M_{f \rightarrow g}^{x y} \approx & -\sum_{n=g, f}\left(\frac{\left\langle\Psi_{g}\left|\mu^{x}\right| \Psi_{n}\right\rangle\left\langle\Psi_{n}\left|\mu^{y}\right| \Psi_{f}\right\rangle}{\Omega_{n g}-\omega_{1 x}-i \epsilon}+\frac{\left\langle\Psi_{g}\left|\mu^{y}\right| \Psi_{n}\right\rangle\left\langle\Psi_{n}\left|\mu^{x}\right| \Psi_{f}\right\rangle}{\Omega_{n g}-\omega_{2 y}-i \epsilon}\right) \\
\approx & -\left\langle\Psi_{g}\left|\mu^{y}\right| \Psi_{f}\right\rangle \frac{\left\langle\Psi_{f}\left|\mu^{x}\right| \Psi_{f}\right\rangle-\left\langle\Psi_{g}\left|\mu^{x}\right| \Psi_{g}\right\rangle}{\omega_{1 x}+i \epsilon} \\
& -\left\langle\Psi_{g}\left|\mu^{x}\right| \Psi_{f}\right\rangle \frac{\left\langle\Psi_{f}\left|\mu^{y}\right| \Psi_{f}\right\rangle-\left\langle\Psi_{g}\left|\mu^{y}\right| \Psi_{g}\right\rangle}{\omega_{2 y}+i \epsilon} .
\end{aligned}
$$

Similarly to the one-photon transition, this 2PA transition also has intramolecular chargetransfer character; its large 2PA moments result from the large one-photon transition dipole moment and the large difference in the dipole moments between the initial and final states. As discussed in Ref. 16, these two quantities are present in the numerators of Eqs. (30) and (31). On the other hand, it is the pole structure (which comes from denominators) of Eqs. (1) and (2) that imparts the resonant character to a RIXS transition. Thus, one can potentially identify two-photon RIXS transitions involving the $\mathrm{XA}_{1}$ and $2 \mathrm{~A}_{1}$ states in pNA for which both the near-resonance (involving intermediate core states) and off-resonance (involving the initial and final valence states) orbital channels are important. Below we provide such an example by considering the $\mathrm{XA}_{1} \rightarrow 2 \mathrm{~A}_{1}$ RIXS transition in pNA for which the incoming photon frequency is tuned to its $\mathrm{XA}_{1} \rightarrow c 6 \mathrm{~B}_{2} \mathrm{C}$-edge resonance at $288.01 \mathrm{eV}$. We compute the $\mathrm{XA}_{1} \rightarrow 2 \mathrm{~A}_{1}$ RIXS cross section using a modified fc-CVS-EOM-EE-CCSD method in which the SOS includes the CVS states plus the initial and the final states, so that the RHS terms in Eqs. (30) and (31) are also incorporated. 


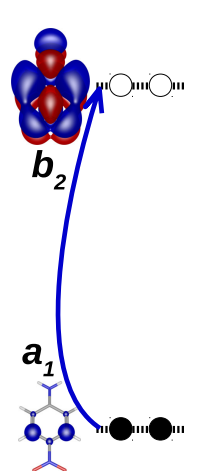

Channel 1

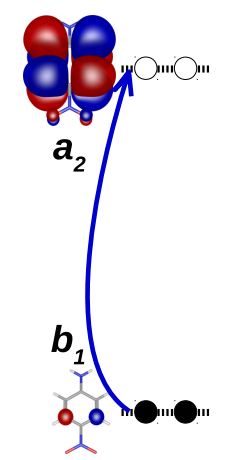

Channel 2

\section{X-ray absorption} $\left(X A_{1} \rightarrow C 6 B_{2}\right)$

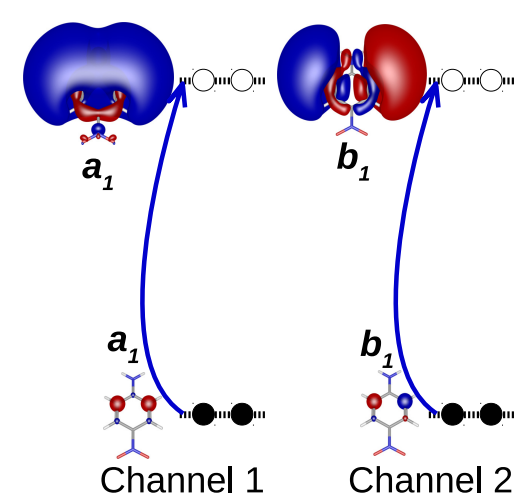

$$
\begin{gathered}
\text { X-ray absorption } \\
\left(\mathrm{XA}_{1} \rightarrow \mathrm{C} 1 \mathrm{~A}_{1}\right)
\end{gathered}
$$

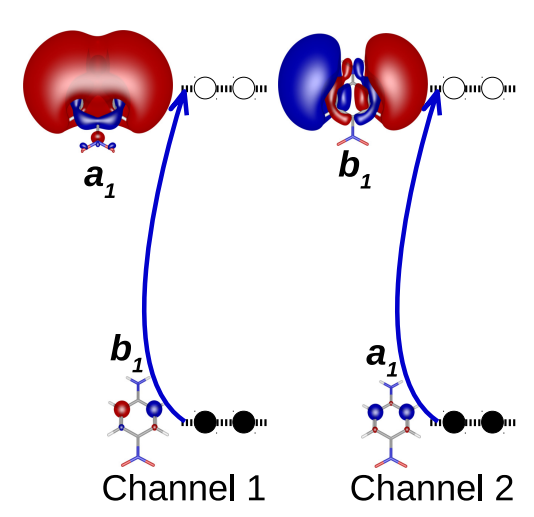

$$
\mathrm{X} \text {-ray absorption }
$$$$
\left(\mathrm{XA}_{1} \rightarrow \mathrm{C1B} \mathrm{B}_{1}\right)
$$

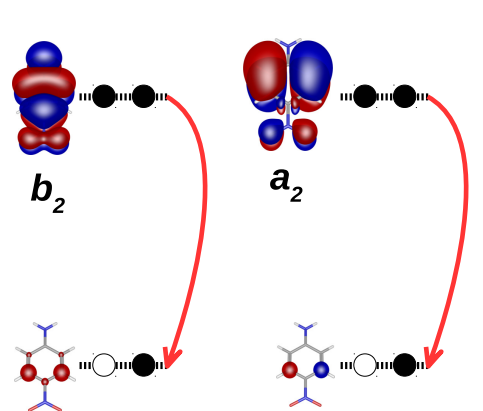

Channel 1 Channel 2

X-ray emission

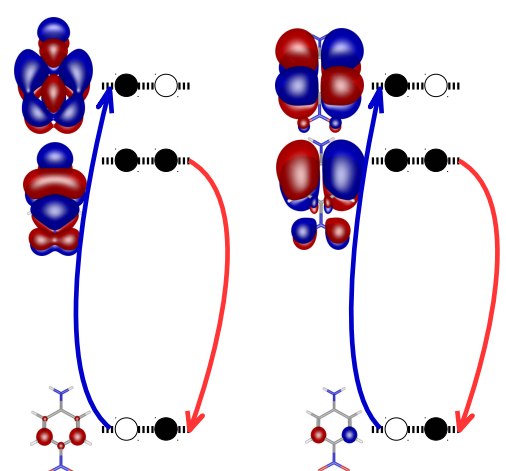

Channel 1 Channel 2

$$
\text { RIXS }
$$

(3-states model)

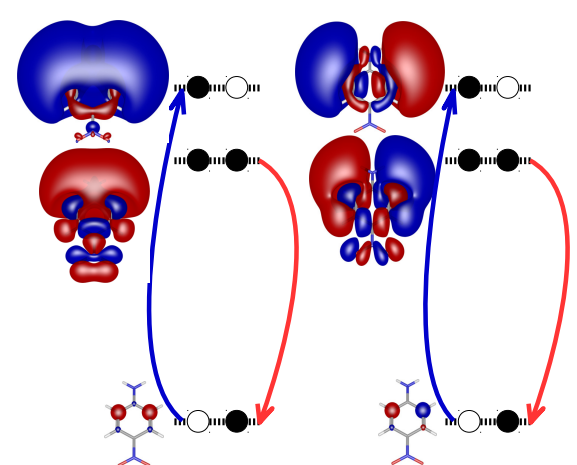

Channel 1

Channel 2

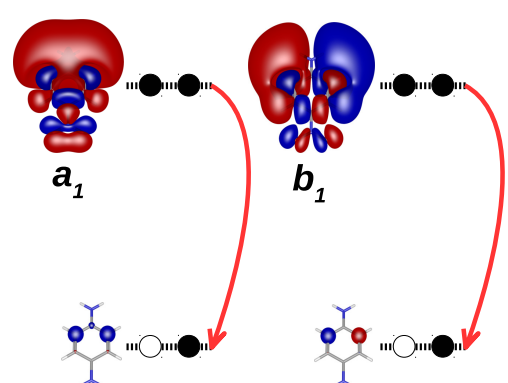

Channel 1 Channel 2

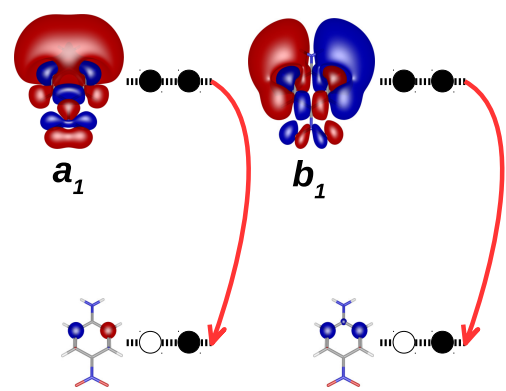

Channel 1

X-ray emission

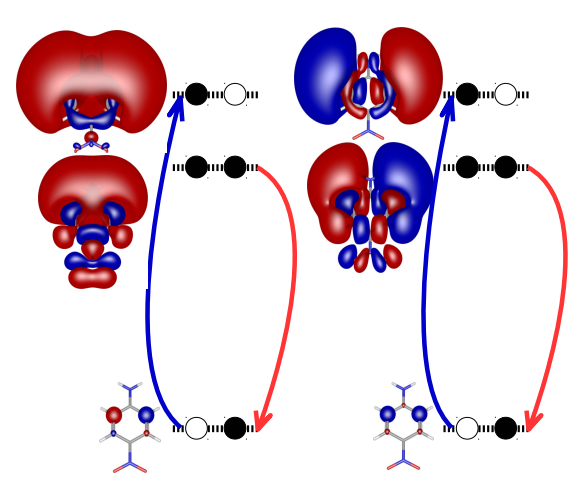

Channel 1
RIXS

(3-states model)

$\mathrm{X}$-ray emission

RIXS

(3-states model)

FIG. 9: NTO analysis of the $\mathrm{XA}_{1} \rightarrow 2 \mathrm{~A}_{1}$ RIXS transition in pare-nitroaniline using the three-states model involving the core-excited (top panel) $\mathrm{c} \mathrm{B}_{2}$ state, (middle panel) $\mathrm{c} \mathrm{A}_{1}$ state, and (bottom panel) $\mathrm{c} 1 \mathrm{~B}_{1}$ state. 
The $\mathrm{XA}_{1} \rightarrow \mathrm{c} 6 \mathrm{~B}_{2}$ core excitation is dark due to symmetry, but it is nearly degenerate with the bright (near-)degenerate $\mathrm{XA}_{1} \rightarrow \mathrm{c} \mathrm{A}_{1}$ and $\mathrm{XA}_{1} \rightarrow \mathrm{c} 1 \mathrm{~B}_{1}$ transitions at $287.96 \mathrm{eV}$ (see the SI). The NTO analysis for $\mathrm{XA}_{1} \rightarrow c 6 \mathrm{~B}_{2}$ core excitation given in the SI and Fig. 9 reveals its two dominant orbital transitions: $c a_{1} \rightarrow b_{2}$ and $c b_{1} \rightarrow a_{2}$. From the NTO analyses of the $\mathrm{XA}_{1} \rightarrow \mathrm{c} 6 \mathrm{~B}_{2}$ x-ray absorption and $\mathrm{c} 6 \mathrm{~B}_{2} \rightarrow 2 \mathrm{~A}_{1}$ x-ray emission, the three-states model suggests the $b_{2} \rightarrow c a_{1} \rightarrow b_{2}$ near-resonance channel should dominate for the $\gamma^{y, \operatorname{Im}}$ and $\tilde{\gamma}^{y, \operatorname{Im}}$ 1PTDMs.

For this RIXS transition, the $M^{x x}, M^{y y}$, and $M^{z z}$ RIXS moments are comparable. $M^{y y, I m}$ and $M^{x x, I m}$ have larger magnitudes, indicating that the near-resonance channels along the $y$ and $x$ axis have the largest contribution. The NTO analyses of $\gamma^{y}$ and $\tilde{\gamma}^{y}$ RIXS 1PTDMs are given in the SI. The near-resonance mechanism of electronic density transfer in the inelastic scattering obtained from analyzing $\gamma^{y, \operatorname{Im}}$ and $\tilde{\gamma}^{y, \operatorname{Im}}$ is not what is expected from the threestates model (Fig. 10); the intermediate core orbitals obtained are a linear combination of the six $1 s_{C}$ molecular orbitals. This reflects that the SOS resonant term with $\Omega_{n g}=\omega_{1}$ does not provide the dominant contribution to the RIXS moment. In fact, the damped contributions from other off-resonance terms that are collected in the $\gamma^{y, \operatorname{Im}}$ and $\tilde{\gamma}^{y, \operatorname{Im}} 1$ PTDMs contribute

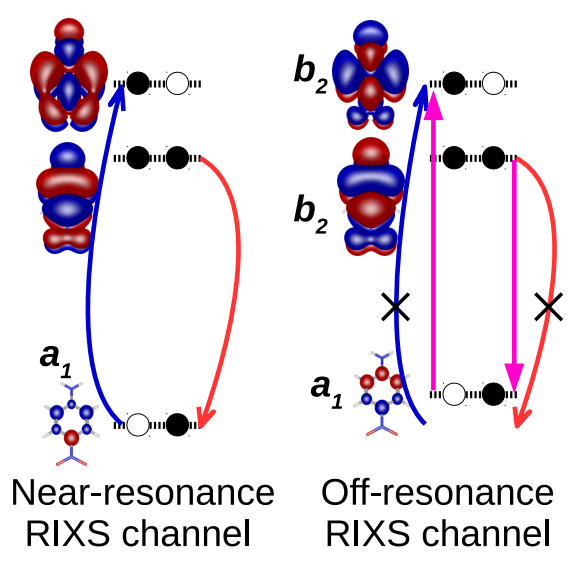

FIG. 10: NTO analysis of the real and imaginary $\gamma^{y}$ and $\tilde{\gamma}^{y} 1 \mathrm{PTDMs}$ for the $\mathrm{XA}_{1} \rightarrow 2 \mathrm{~A}_{1}$ RIXS transition in para-nitroaniline. Here, the off-resonance channel is dominant and the near-resonance channel is not the one predicted by the three-states model. For both channels, the core-hole NTOs are given as a linear combination of the six $1 s_{C}$ molecular orbitals as the large numerators of some of the off-resonance SOS terms make their contribution larger than that of the near-resonant SOS terms even in the imaginary components of these 1PTDMs. 
more than the near-resonance term primarily due to larger transition dipole moments than the ones forming the near-resonance term (i.e., the transition dipole moments for $\mathrm{XA}_{1} \rightarrow$ $\mathrm{c} 6 \mathrm{~B}_{2}$ and $2 \mathrm{~A}_{1} \rightarrow \mathrm{c} 6 \mathrm{~B}_{2}$ transitions). The fact that the off-resonance terms are dominant is also reflected in the larger norms of the $\gamma^{y, \operatorname{Re}}$ and $\tilde{\gamma}^{y, \operatorname{Re}} 1 \mathrm{PTDMs}$ than the $\gamma^{y, \operatorname{Im}}$ and $\tilde{\gamma}^{y, \operatorname{Im}}$ 1PTDMs $\left(v^{y, \operatorname{Im}}=0.03\right)$. Further, the RMS electron-hole distances $\left(d_{e x c}^{y}\right)$ computed for these components of the RIXS transition $(\approx 2.4 \AA)$ is smaller than the $3.6 \AA$ computed for the one-photon $\mathrm{XA}_{1} \rightarrow 2 \mathrm{~A}_{1}$ transition (see the $\mathrm{SI}$ ), highlighting the local character of the RIXS transition along the $y$ direction.

Since the $\mathrm{XA}_{1} \rightarrow \mathrm{c} 6 \mathrm{~B}_{2}$ core excitation is nearly degenerate with $\mathrm{XA}_{1} \rightarrow \mathrm{c} 1 \mathrm{~A}_{1}$ and $\mathrm{XA}_{1} \rightarrow$ $\mathrm{c}_{1} \mathrm{~B}_{1}$ core excitations, the latter two transitions with larger oscillator strengths open nearresonance orbital channels and impact the imaginary $M_{z z}$ and $M_{x x}$ RIXS moments. As shown in Fig. 9, the $c a_{1} \rightarrow a_{1}$ and $c b_{1} \rightarrow b_{1}$ orbital transitions are important in the $\mathrm{XA}_{1} \rightarrow$ $c 1 A_{1}$ core excitation and the $c b_{1} \rightarrow a_{1}$ and $c a_{1} \rightarrow b_{1}$ orbital transitions are important in the $\mathrm{XA}_{1} \rightarrow \mathrm{c}^{1} \mathrm{~B}_{1}$ core excitation. These orbital transitions also dominate the NTO analysis of $\tilde{\gamma}^{z, \operatorname{Im}}$ and $\tilde{\gamma}^{x, \operatorname{Im}} 1 \mathrm{PTDMs}$ consistent with the three-states models constructed with the NTO analyses of these two XAS peaks. Further, the RMS electron-hole distances for these 1PTDMs are smaller than the one computed for the one-photon $\mathrm{XA}_{1} \rightarrow 2 \mathrm{~A}_{1}$ transition, indicating that these near-resonance channels are local and confined to the respective active core-hole orbitals.

For the $M_{x x}$ RIXS moments, the imaginary components are larger than the respective real
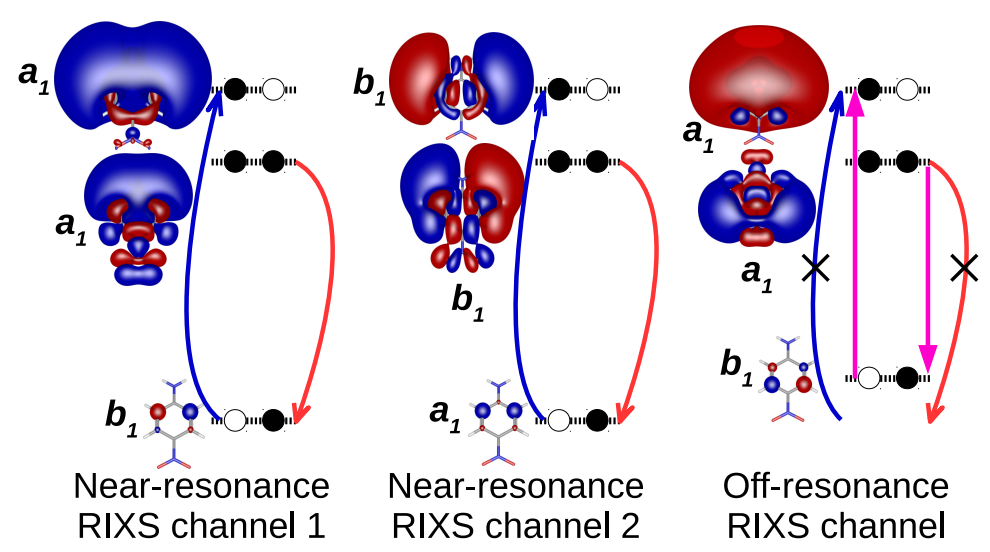

FIG. 11: NTO analysis of the real and imaginary $\gamma^{x}$ and $\tilde{\gamma}^{x} 1 \mathrm{PTDMs}$ for the $\mathrm{XA}_{1} \rightarrow 2 \mathrm{~A}_{1}$ RIXS transition in para-nitroaniline. Here, the near-resonance channel 1 is dominant. The two nearresonance channels are also consistent with the ones predicted by the three-states model. 


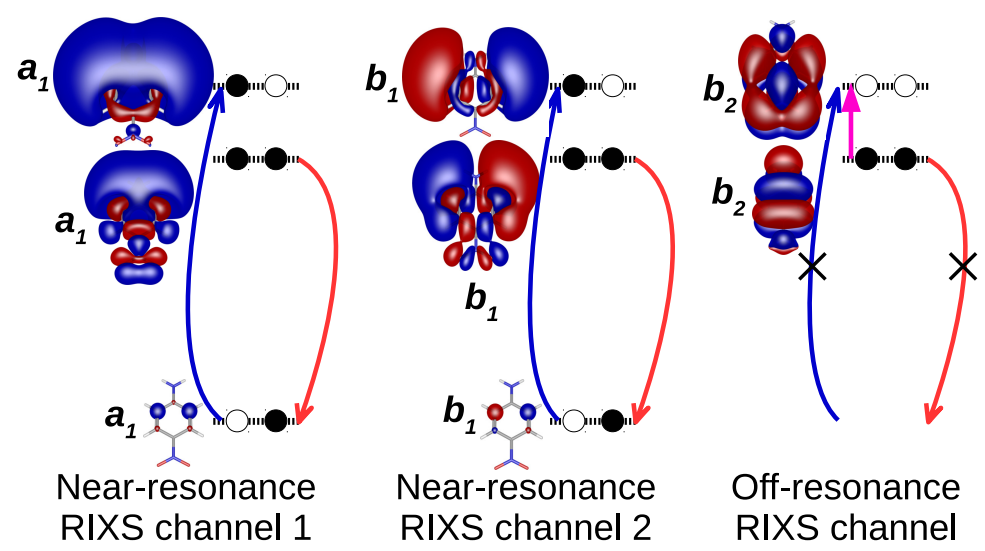

FIG. 12: NTO analysis of the real and imaginary $\gamma^{z}$ and $\tilde{\gamma}^{z}$ 1PTDMs for the $\mathrm{XA}_{1} \rightarrow 2 \mathrm{~A}_{1}$ RIXS transition in para-nitroaniline. Here, the off-resonance channel is dominant and features the same $\pi \rightarrow \pi^{*}$ intramolecular charge-transfer channel that characterizes the one-photon $\mathrm{XA}_{1} \rightarrow 2 \mathrm{~A}_{1}$ transition. The near-resonance channel is the one predicted by the three-states model.

components $\left(v^{x, \operatorname{Im}}=0.71\right)$, consistent with larger norm for the $\gamma^{x, \operatorname{Im}}$ and $\tilde{\gamma}^{x, \operatorname{Im}}$ 1PTDMs than the respective $\gamma^{x, \operatorname{Re}}$ and $\tilde{\gamma}^{x, \operatorname{Re}} 1 \mathrm{PTDMs}$ (Fig. 11). On the other hand, the real components are larger than the imaginary components for the $M_{z z}$ moments $\left(v^{z, \operatorname{Im}}=0.01\right)$, consistent with the larger norms for $\gamma^{z, \operatorname{Re}}$ and $\tilde{\gamma}^{z, \operatorname{Re}} 1$ PTDMs than those for $\gamma^{z, \operatorname{Im}}$ and $\tilde{\gamma}^{z, \operatorname{Im}} 1$ PTDMs. The NTO analyses of the $\gamma^{z, \operatorname{Re}}$ and $\tilde{\gamma}^{z, \text { Re }} 1$ PTDMs show that the $4 b_{2} \rightarrow 5 b_{2}$ transition is the significant off-resonance RIXS channel, highlighting that this two-photon process has some intramolecular charge-transfer character (Fig. 12). This is further supported by the larger RMS electron-hole distances for the $\gamma^{z, \text { Re }}$ and $\tilde{\gamma}^{z, \text { Re }}$ 1PTDMs than the other RIXS 1PTDMs and comparable to the RMS electron-hole distance for the one-photon $\mathrm{XA}_{1} \rightarrow$ $2 \mathrm{~A}_{1}$ transition. This intramolecular $\pi \rightarrow \pi^{*}$ charge-transfer channel is, however, not the dominant off-resonance channel in the overall RIXS process as $\Upsilon^{z, \operatorname{Re}}<\Upsilon^{y, \text { Re}}$, even though the overall character of this RIXS transition is not resonant $\left(\Upsilon^{\operatorname{Im}}=0.03\right)$.

\section{Wave-function analysis of RIXS transitions in 4-amino-4'-nitrostilbene}

Similar to pNA, 4-amino-4'-nitrostilbene (4A4NS) is a push-pull chromophore. Its onephoton $\mathrm{XA}^{\prime} \rightarrow 2 \mathrm{~A}^{\prime}$ transition has an even larger oscillator strength than that in pNA, with strong intramolecular charge-transfer character $\left(f=1.22, \Delta \mu^{x}=5.0\right.$ a.u.; see NTO analysis of this transition in the SI). For this molecule, we pick the XA' $\rightarrow 2 \mathrm{~A}^{\prime}$ RIXS transition and 
the incoming photon frequency that is resonant with its lowest N-edge XAS peak $\left(\mathrm{XA}^{\prime} \rightarrow\right.$ c1A') computed at $404.01 \mathrm{eV}$. As in the pNA example discussed above, here we also use the modified fc-CVS-EOM-EE-CCSD method. For the XA' $\rightarrow$ 2A' RIXS transition, only the real $M_{x x}$ moments and the real and imaginary $M_{z z}$ moments are important. The NTO analysis in Fig. 13 of the $\gamma^{x \text {,Re }}$ and $\tilde{\gamma}^{x, \text { Re }}$ RIXS 1PTDMs identifies its dominant off-resonance intramolecular charge-transfer channel $\left(v^{x, \operatorname{Re}}=1.00\right)$, which also describes the one-photon $\mathrm{XA} \rightarrow 2 \mathrm{~A}^{\prime}$ transition. The NTO analyses of the real and imaginary components of $\gamma^{z}$ and $\tilde{\gamma}^{z}$ 1PTDMs show similar orbitals (see the SI) with the norms of the real 1PTDMs larger than the imaginary 1 PTDMs $\left(v^{z, \operatorname{Im}}=0.28\right)$. This indicates that important orbital channels along the $z$ axis are off-resonance, originating from the large numerators in the SOS off-resonance terms, which also dominate the imaginary 1PTDMs. This is not surprising because the lowest N-edge XAS peak (at which the incoming photon energy is tuned) is separated by more than $1 \mathrm{eV}$ from other XAS transitions. Clearly, a few-core-excited-states model would be inadequate for this transition, dominated by off-resonance channels, in particular, the intramolecular charge-transfer channel-unlike in the case of pNA - with $\Upsilon^{x, \text { Re }}$ equal to 0.50. This example provides another illustration of the merits of a fully analytic approach for characterizing RIXS transitions.
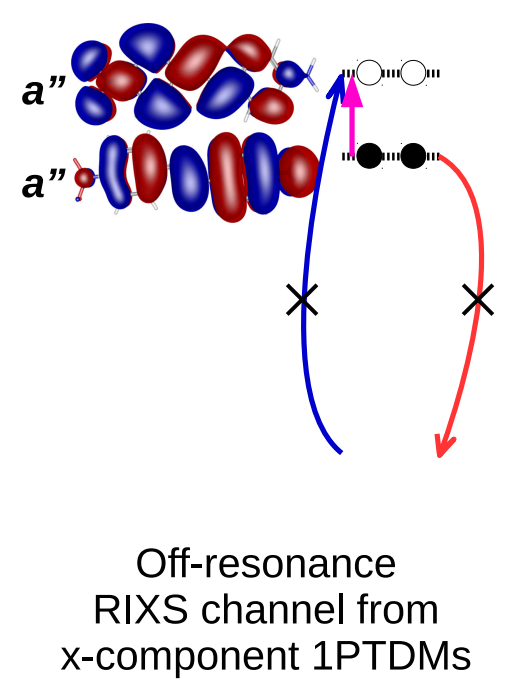

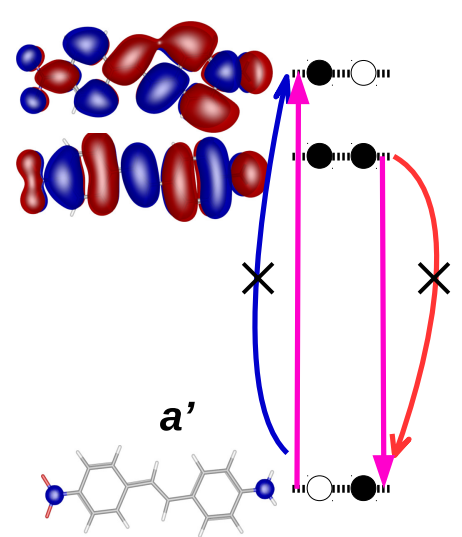

Off-resonance RIXS channel from z-component 1PTDMs

FIG. 13: NTO analysis of the $x$ - and $z$-component 1PTDMs for the XA' $\rightarrow 2 \mathrm{~A}^{\prime}$ RIXS transition in 4-amino-4'-nitrostilbene. Here, the off-resonance channels are dominant. The three-states model predicts near-resonance channels; thus, it is inadequate for this transition. 


\section{CONCLUSION}

We presented a novel black-box approach for deriving the molecular orbital picture of RIXS transitions based on the corresponding response 1PTDMs and their NTOs. This is the first example of the generalization of the concept of NTOs to nonlinear x-ray processes. This new tool for analyzing RIXS transitions relies on the rigorous and compact formalism of the response 1PTDMs based on damped response theory. Not only do the NTOs computed with this approach facilitate the visualization of RIXS transitions, 1PTDMs are also useful for computing physical quantities related to the spatial extent of the RIXS transitions such as the average electron-hole separation. This approach is superior to the traditional few-states approach, which relies on computing few intermediate core-excited states for a qualitative orbital picture of RIXS. The few-states approach inherently suffers from the arbitrariness of the choice of intermediate states and potential loss of accuracy. We demonstrate the utility of the new analysis tool by calculating the orbital picture of RIXS transitions in the $\mathrm{OH}$ radical, benzene, pNA, and 4A4NS molecules. The RIXS transitions in the latter two systems have significant contributions from off-resonance orbital channels, which are difficult to capture with the few-states models, illustrating the merits of rigorous analytic approaches for analyzing RIXS transitions. For chromophores in complex environments, $a b$ initio methods augmented with our analysis tool can help elucidate the role of molecular structure and intermolecular interactions on the RIXS spectra and can provide a rigorous characterization of experimental RIXS spectra.

\section{Supplementary Material}

This document contains: tabulated NTO analysis for valence absorption, x-ray absorption, x-ray emission, and RIXS transitions; relevant Cartesian coordinates; and basis sets used in our calculations.

\section{Acknowledgments}

This work was supported by the U.S. National Science Foundation (No. CHE-1856342). 


\section{Conflicts of interest}

A.I.K. is the President and a part-owner of Q-Chem, Inc.

1 Gel'mukhanov, F.; Ågren, H. Resonant X-ray raman scattering Phys. Rep. 1999, 312, 87-330.

2 Butorin, S.M. Resonant inelastic X-ray scattering as a probe of optical scale excitations in strongly electron-correlated systems: quasi-localized view J. Electron. Spectrosc. Relat. Phenom. 2000, 110-111, 213-233.

3 deGroot, F. High-resolution X-ray emission and X-ray absorption spectroscopy Chem. Rev. 2001, 101, 1779-1808.

4 Kotani, A.; Shin, S. Resonant inelastic x-ray scattering spectra for electrons in solids Rev. Mod. Phys. 2001, 73, 203-246.

5 Ament, L.J.P.; van Veenendaal, M.; Devereaux, T. P.; Hill, J.P.; van den Brink, J. Resonant inelastic x-ray scattering studies of elementary excitations Rev. Mod. Phys. 2011, 83, 705-767.

6 Schmitt, T.; deGroot, F.M.F.; Rubensson, J. Prospects of high-resolution resonant X-ray inelastic scattering studies on solid materials, liquids and gases at diffraction-limited storage rings J. Synchrotron Rad. 2014, 21, 1065-1076.

7 Eckert, S.; Norell, J.; Miedema, P.S.; Beye, M.; Fondell, M.; Quevedo, W.; Kennedy, B.; Hantschmann, M.; Pietzsch, A.; Van Kuiken, B.E.; Ross, M.; Minitti, M.P.; Moeller, S.P.; Schlotter, W.F.; Khalil, M.; Odelius, M.; Föhlisch, A. Ultrafast independent N-H and N-C bond deformation investigated with resonant inelastic Xray scattering Phys. Rev. Lett. 2017, $56,6088-6092$.

8 Kjellsson, L.; Nanda, K.; Rubensson, J.-E.; Doumy, G.; Southworth, S. H.; Ho, P. J.; March, A. M.; Al Haddad, A.; Kumagai, Y.; Tu, M.-F.; Debnath, T.; Bin Mohd Yusof, M. S.; Arnold, C.; Schlotter, W. F.; Moeller, S.; Coslovich, G.; Koralek, J. D.; Minitti, M. P.; Vidal, M. L.; Simon, M.; Santra, R.; Loh, Z.-H.; Coriani, S.; Krylov, A. I.; Young, L. RIXS reveals hidden local transitions of the aqueous $\mathrm{OH}$ radical Phys. Rev. Lett. 2020; submitted; https://arxiv.org/abs/2003.03909.

9 Luzanov, A. V.; Sukhorukov, A. A.; Umanskii, V. E. Application of transition density matrix for analysis of excited states Theor. Exp. Chem. 1976, 10, 354-361. 
10 Luzanov, A. V.; Pedash, V. F. Interpretation of excited states using charge-transfer number Theor. Exp. Chem. 1979, 15, 338-341.

11 Head-Gordon, M.; Grana, A. M.; Maurice, D.; White, C. A. Analysis of electronic transitions as the difference of electron attachment and detachment densities J. Phys. Chem. 1995, 99, $14261-14270$.

12 Plasser, F.; Wormit, M.; Dreuw, A. New tools for the systematic analysis and visualization of electronic excitations. I. Formalism J. Chem. Phys. 2014, 141, 024106-13.

13 Plasser, F.; Bäppler, S. A.; Wormit, M.; Dreuw, A. New tools for the systematic analysis and visualization of electronic excitations. II. Applications J. Chem. Phys. 2014, 141, 024107-12.

14 Bäppler, S. A.; Plasser, F.; Wormit, M.; Dreuw, A. Exciton analysis of many-body wave functions: Bridging the gap between the quasiparticle and molecular orbital pictures Phys. Rev. A 2014, 90, 052521.

15 Mewes, S.; Plasser, F.; Krylov, A. I.; Dreuw, A. Benchmarking excited-state calculations using exciton properties J. Chem. Theory Comput. 2018, 14, 710-725.

16 Nanda, K.; Krylov, A. I. Visualizing the contributions of virtual states to two-photon absorption cross-sections by natural transition orbitals of response transition density matrices J. Phys. Chem. Lett. 2017, 8, 3256-3265.

17 Skomorowski, W.; Krylov, A. I. Real and imaginary excitons: Making sense of resonance wavefunctions by using reduced state and transition density matrices J. Phys. Chem. Lett. 2018, 9, $4101-4108$.

18 Pokhilko, P.; Krylov, A. I. Quantitative El-Sayed rules for many-body wavefunctions from spinless transition density matrices J. Phys. Chem. Lett. 2019, 10, 4857-4862.

19 Rehn, D.R.; Dreuw, A.; Norman, P. Resonant inelastic Xray scattering amplitudes and cross sections in the algebraic diagrammatic construction/intermediate state representation (ADC/ISR) approach J. Chem. Theory Comput. 2017, 13, 5552-5559.

20 Faber, R.; Coriani, S. Resonant inelastic X-ray scattering and nonesonant X-ray emission spectra from coupled-cluster (damped) response theory J. Chem. Theory Comput. 2019, 15, 520-528.

21 Nanda, K.; Vidal, M. L.; Faber, R.; Coriani, S.; Krylov, A. I. How to stay out of trouble in RIXS calculations within the equation-of-motion coupled-cluster damped response theory framework? Safe hitchhiking in the excitation manifold by means of core-valence separation Phys. Chem. Chem. Phys. 2020, 22, 2629-2641. 
22 Faber, R.; Coriani, S. Core-valence-separated coupled-cluster-singles-and-doubles complexpolarization-propagator approach to X-ray spectroscopies Phys. Chem. Chem. Phys. 2020, 22, $2642-2647$.

23 Lundberg, M.; Kroll, T.; DeBeer, S.; Bergmann, U.; Wilson, S. A.; Glatzel, P.; Nordlund, D.; Hedman, B.; Hodgson, K. O.; Solomon, E. I. Metalligand covalency of iron complexes from high-resolution resonant inelastic xray scattering J. Am. Chem. Soc. 2013, 135, 17121-17134.

24 Maganas, D.; Kristiansen, P.; Duda, L.-C.; Knop-Gericke, A.; DeBeer, S.; Schlögl, R.; Neese, F. Combined experimental and ab initio multireference configuration interaction study of the resonant inelastic Xray scattering spectrum of $\mathrm{CO}_{2}$ J. Phys. Chem. C 2014, 118, 20163-20175.

25 Ertan, E.; Savchenko, V.; Ignatova, N.; Vaz da Cruz, V.; Couto, R. C.; Eckert, S.; Fondell, M.; Dantz, M.; Kennedy, B.; Schmitt, T.; Pietzsch, A.; Föhlisch, A.; Gel'mukhanov, F.; Odelius, M.; Kimberg, V. Ultrafast dissociation features in RIXS spectra of the water molecule Phys. Chem. Chem. Phys. 2018, 20, 14384-14397.

26 Helgaker, T.; Coriani, S.; Jørgensen, P.; Kristensen, K.; Olsen, J.; Ruud, K. Recent advances in wave function-based methods of molecular-property calculations Chem. Rev. 2012, 112, 543631.

27 Norman, P.; Dreuw, A. Simulating X-ray spectroscopies and calculating core-excited states of molecules Chem. Rev. 2018, 118, 7208-7248.

28 Nanda, K. D.; Krylov, A. I. Two-photon absorption cross sections within equation-of-motion coupled-cluster formalism using resolution-of-the-identity and Cholesky decomposition representations: Theory, implementation, and benchmarks J. Chem. Phys. 2015, 142, 064118.

29 Nanda, K. D.; Krylov, A. I. Static polarizabilities for excited states within the spin-conserving and spin-flipping equation-of-motion coupled-cluster singles and doubles formalism: Theory, implementation, and benchmarks J. Chem. Phys. 2016, 145, 204116.

30 Nanda, K.; Krylov, A. I.; Gauss, J. COMMUNICATION: The pole structure of the dynamical polarizability tensor in equation-of-motion coupled-cluster theory J. Chem. Phys. 2018, 149, 141101.

31 Nanda, K.; Krylov, A. I. The effect of polarizable environment on two-photon absorption cross sections characterized by the equation-of-motion coupled-cluster singles and doubles method combined with the effective fragment potential approach J. Chem. Phys. 2018, 149, 164109.

32 Plasser, F. Entanglement entropy of electronic excitations J. Chem. Phys. 2016, 144, 194107. 
33 Tretiak, S.; Mukamel, S. Density matrix analysis and simulation of electronic excitations in conjugated and aggregated molecules Chem. Rev. 2002, 102, 3171.

34 Sun, M.; Chen, J.; Xu, H. Visualizations of transition dipoles, charge transfer, and electron-hole coherence on electronic state transitions between excited states for two-photon absorption $J$. Chem. Phys. 2008, 128, 064106.

35 Shao, Y.; Gan, Z.; Epifanovsky, E.; Gilbert, A.T.B.; Wormit, M.; Kussmann, J.; Lange, A.W.; Behn, A.; Deng, J.; Feng, X., et al. Advances in molecular quantum chemistry contained in the Q-Chem 4 program package Mol. Phys. 2015, 113, 184-215.

36 Krylov, A. I.; Gill, P. M. W. Q-Chem: An engine for innovation WIREs: Comput. Mol. Sci. 2013, 3, 317-326.

37 Vidal, M. L.; Feng, X.; Epifanovski, E.; Krylov, A. I.; Coriani, S. A new and efficient equationof-motion coupled-cluster framework for core-excited and core-ionized states J. Chem. Theory Comput. 2019, 15, 3117-3133.

38 Sarangi, R.; Vidal, M. L.; Coriani, S.; Krylov, A. I. On the basis set selection for calculations of core-level states: Different strategies to balance cost and accuracy Mol. Phys. 2020; submitted; https://doi.org/10.26434/chemrxiv.12079587.v1.

39 Allouche, A. Gabedit-a graphical user interface for computational chemistry softwares J. Comput. Chem. 2011, 32, 174-182.

40 IQmol molecular viewer. Gilbert, A.T.B. http://iqmol.org.

41 Loh, Z.-H.; Doumy, G.; Arnold, C.; Kjellsson, L.; Southworth, S. H.; Al Haddad, A.; Kumagai, Y.; Tu, M.-F.; Ho, P. J.; March, A. M.; Schaller, R. D.; Bin Mohd Yusof, M. S.; Debnath, T.; Simon, M.; Welsch, R.; Inhester, L.; Khalili, K.; Nanda, K.; Krylov, A. I.; Moeller, S.; Coslovich, G.; Koralek, J.; Minitti, M. P.; Schlotter, W. F.; Rubensson, J.-E.; Santra, R.; Young, L. Observation of the fastest chemical processes in the radiolysis of water Science 2020, 367, 179-182.

42 Hitchcock, A. P.; Pocock, M.; Brion, C. E.; Banna, M. S.; Frost, D. C.; McDowell, C. A.; Wallbank, B. Inner shell excitation and ionization of the monohalobenzenes J. Elec. Spect. and Rel. Phen. 1978, 13, 345-360.

43 Horsley, J. A.; Stöhr, J.; Hitchcock, A. P.; Newbury, D. C.; Johnson, A. L.; Sette, F. Resonances in the K-shell excitation spectra of benzene and pyridine: Gas phase, solid, and chemisorbed states J. Chem. Phys. 1985, 83, 6099-6107. 
44 Schwarz, W. H. E.; Chang, T. C.; Seeger, U.; Hwang, K. H. Core excitations of symmetrical aromatic molecules. Specific correlations in the valence shell and localization in the core shells Chem. Phys. 1987, 117, 73-89.

45 Ma, Y.; Sette, F.; Meigs, G.; Modesti, S.; Chen, C. T. Breaking of ground-state symmetry in core-excited ethylene and benzene Phys. Rev. Lett. 1989, 63, 2044.

46 Menzel, D.; Rocker, G.; Steinrück, H.-P.; Heimann, D. Coulmanand P. A.; Huber, W.; Zebisch, P.; Lloyd, D. R. Core excitation, decay, and fragmentation in solid benzene as studied by xray absorption, resonant Auger, and photon stimulated desorption J. Chem. Phys. 1992, 96, 1724 .

47 Rennie, E. E.; Kempgens, B.; Köppe, H. M.; Hergenhahn, U.; Feldhaus, J.; Itchkawitz, B. S.; Kilcoyne, A. L. D.; Kivimäki, A.; Maier, K.; Piancastelli, M. N.; Polcik, M.; Rüdel, A.; Bradshaw, A. M. A comprehensive photoabsorption, photoionization, and shake-up excitation study of the C1s cross section of benzene J. Chem. Phys. 2000, 113, 7362-7375.

48 Püttner, R.; Kolczewski, C.; Martins, M.; Schlachter, A. S.; Snell, G.; Sant'Anna, M.; Viefhaus, J.; Hermann, K.; Kaindl, G. The C 1s NEXAFS spectrum of benzene below threshold: Rydberg or valence character of the unoccupied $\sigma$-type orbitals Chem. Phys. Lett. 2004, 393, 361-366.

49 Kolczewski, C.; Püttner, R.; Martins, M.; Schlachter, A. S.; Snell, G.; Sant'Anna, M. M.; Hermann, K.; Kaindl, G. Spectroscopic analysis of small organic molecules: A comprehensive near-edge x-ray-absorption fine-structure study of $\mathrm{C}_{6}$-ring-containing molecules $J$. Chem. Phys. 2006, 124, 034302.

50 Khalil, O.S.; Seliskar, C.J.; McGlynn, S.P. Electronic spectroscopy of highly-polar aromatics. II. Luminescence of nitroanilnes J. Chem. Phys. 1973, 58, 1607-1612.

51 Carsey, T.P.; Findley, G.L.; McGlynn, S.P. Systematics in the electronic spectra of polar molecules. 1. Para-disubstituted benzenes J. Am. Chem. Soc. 1979, 101, 4502-4510.

52 Kovalenko, S.A.; Schanz, R.; Farztdinov, V.M.; Hennig, H.; Ernsting, N.P. Femtosecond relaxation of photoexcited para-nitroaniline: solvation, charge transfer, internal conversion and cooling Chem. Phys. Lett. 2000, 323, 312-322.

53 Slipchenko, L. V. Solvation of the excited states of chromophores in polarizable environment: Orbital relaxation versus polarization J. Phys. Chem. A 2010, 114, 8824-8830. 